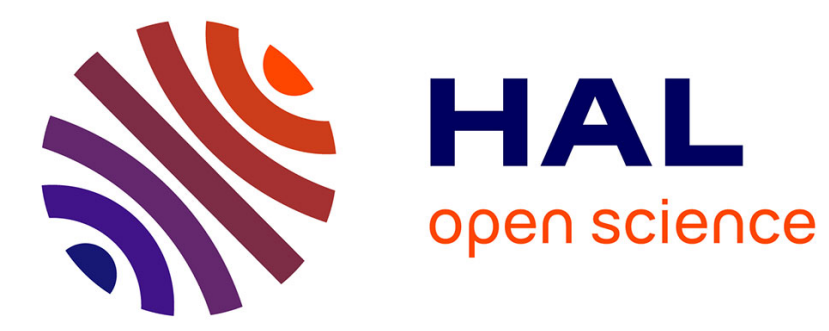

\title{
Les familles ouvrières face au devenir de leurs enfants
}

Tristan Poullaouec

\section{To cite this version:}

Tristan Poullaouec. Les familles ouvrières face au devenir de leurs enfants. Economie et Statistique / Economics and Statistics, 2004, 10.3406/estat.2004.7272 . halshs-01214784

\section{HAL Id: halshs-01214784 \\ https://shs.hal.science/halshs-01214784}

Submitted on 13 Oct 2015

HAL is a multi-disciplinary open access archive for the deposit and dissemination of scientific research documents, whether they are published or not. The documents may come from teaching and research institutions in France or abroad, or from public or private research centers.
L'archive ouverte pluridisciplinaire HAL, est destinée au dépôt et à la diffusion de documents scientifiques de niveau recherche, publiés ou non, émanant des établissements d'enseignement et de recherche français ou étrangers, des laboratoires publics ou privés. 


\title{
Les familles ouvrières face au devenir de leurs enfants
}

\author{
Tristan Poullaouec*
}

Si la plupart des ouvriers souhaitent aujourd'hui que leurs enfants échappent aux emplois d'exécution, ils espèrent aussi souvent qu'ils exercent le métier qui leur plaira, pourvu qu'ils réussissent leurs études. Intégrant le nouveau rôle des titres scolaires dans le modelage des trajectoires sociales, les ouvriers placent de plus en plus l'école au centre de leurs stratégies éducatives. Enjeu incontournable pour l'avenir de la lignée, la prolongation des études s'inscrit dans une dynamique intergénérationnelle : l'espoir des ouvriers que leurs enfants réalisent par la voie scolaire ce dont ils ont rêvé pour eux-mêmes témoigne ici d'une reprise des aspirations d'une génération à l'autre.

Mais si les diplômes sont de plus en plus nécessaires pour garantir une bonne insertion, ils sont aussi de moins en moins suffisants, ce qui peut conduire les parents à s'investir dans les stratégies d'accès à l'emploi de leurs enfants. L'implication des parents pour permettre aux enfants d'obtenir un emploi perdure dans le monde ouvrier. Un quart des enfants d'ouvriers nés entre la fin de la guerre et le milieu des années 1970 ont ainsi reçu une aide de leurs parents pour accéder à un emploi. La mobilisation des relations des parents donne des résultats ambivalents : si elle permet peut-être d'éviter le chômage aux enfants les moins diplômés, elle augmente les risques de devenir ouvrier et ne modifie pas significativement les chances d'atteindre des positions de cadre supérieur ou de profession intermédiaire.

Sans contester les clivages entre générations, cet article les relativise en soulignant qu'au-delà des tensions ou incompréhensions entre parents et enfants, les aspirations des uns et des autres s'inscrivent dans une même histoire familiale où les deux générations se rejoignent dans l'appropriation des enjeux scolaires.

* Tristan Poullaouec appartient au Laboratoire Printemps du CNRS, Université de Versailles-Saint-Quentin-en-Yvelines. Les noms et dates entre parenthèses renvoient à la bibliographie en fin d'article 
$\mathbf{S}$ ecret ou avoué, calculé ou simplement rêvé, l'objectif de la plupart des parents est de voir leurs enfants atteindre une position sociale équivalente, sinon supérieure à la leur. Si évident et commun qu'il paraisse, l'enjeu revêt cependant une signification historique particulière à bien des égards dans les familles ouvrières. Aujourd'hui peut-être plus encore qu'hier, la peur du déclassement, voire le «spectre» de la délinquance des enfants hante les ouvriers (1). La condition ouvrière ne semble plus aussi acceptable pour les ouvriers eux-mêmes, a fortiori pour leurs enfants, et les aspirations à s'en évader, le désir que les enfants rejoignent d'autres positions au sein du salariat non ouvrier sont devenus très forts. Sur fond de crise du monde ouvrier, la transmission d'un certain héritage ouvrier serait donc devenue problématique (Beaud et Pialoux, 1999). Qu'un de leurs enfants s'engage dans la même activité professionnelle qu'eux ne satisferait plus désormais qu'une faible minorité d'ouvriers (2). La disqualification profonde et massive des destins ouvriers semble bien être au cœur du problème.

L'analyse des modalités de constitution des générations ouvrières, au-delà de la seule sphère familiale, permet de mieux comprendre cette crise de reproduction du groupe ouvrier. En s'en tenant ici aux conditions de la scolarisation et de la transition professionnelle, on mesure bien le contraste entre les biographies collectives des uns et des autres selon leur génération (3). Les jeunes d'origine ouvrière nés dans les années 1970 ont ainsi connu la généralisation de l'accès au collège puis au lycée et ont fait leurs premiers pas sur le marché du travail dans les années 1990, dans un contexte marqué par la dégradation des conditions d'accès à l'emploi, le chômage de masse, le blocage des salaires, etc. Leurs parents, nés pour la plupart dans les années 1940, souvent d'origine ouvrière eux-mêmes, ont rarement prolongé leur scolarité au-delà du primaire, sinon pour préparer des CAP à l'époque valorisés sur le marché du travail, dans des collèges techniques alors scolairement sélectifs. Ils ont commencé leur vie professionnelle dans les années 1960 dans une conjoncture où la pénurie de main-d'œuvre et l'amélioration du niveau de vie leur assuraient un emploi stable et une certaine aisance sans forcément quitter la condition ouvrière (4).

$\mathrm{S}$ 'agissant de comprendre le présent en regard du passé, on peut donc identifier avec profit certains clivages «générationnels » au sein du groupe ouvrier (5) qui modifient les conditions de la transmission. Ce faisant, on ne saurait se contenter de décrire les relations sociales au cœur des lignées ouvrières en soulignant seulement à quel point parents et enfants diffèrent. Le risque est alors grand de ne voir dans les familles ouvrières que la distance entre des générations qui ne se reconnaissent plus dans le miroir qu'elles se tendent réciproquement, comme si les générations en présence étaient devenues complètement étrangères l'une pour l'autre. Nombreux sont ceux qui ont souligné par ailleurs la force, la diversité et la continuité des liens entre générations au sein de la parenté. Si la mise en évidence d'effets de génération et de césures intergénérationnelles ne contredit pas l'importance toujours attestée des solidarités intergénérationnelles, c'est sans doute parce que les rapports qui se nouent entre les générations sont à la fois un produit et un vecteur du changement social (Attias-Donfut, 2000).

Il s'agit ici de montrer comment les transformations de l'institution scolaire et du monde du travail sont intégrées dans les stratégies éducatives familiales et de quelle manière elles se traduisent dans les transmissions intergénérationnelles des familles ouvrières. En premier lieu, on analysera les aspirations professionnelles aujourd'hui formulées par les ouvriers pour leurs enfants et ce qu'elles doivent à la généralisation de la scolarisation de génération en génération. La question de l'évolution de la

1. Comme l'écrivait Michel Verret (1992) : « [...] quand le déclassement touche la dernière classe, la distance est petite de la soustraction de classe à la soustraction au monde ".

2. Baudelot et Gollac, 2003, pp. 134-135: « [...] les catégories qui se déclarent les moins heureuses à l'idée que leurs enfants suivent leurs traces sont majoritairement constituées de travailleurs manuels (ouvriers, chauffeurs) ainsi que des personnels d'exécution du secteur tertiaire, employés et services aux particuliers ".

3. Que ces générations soient loin d'être homogènes, y compris à l'intérieur d'un même groupe social, ne suffit pas à invalider toute caractérisation des cohortes les unes par rapport aux autres comme des générations distinctes. Quelles que soient leurs différences par ailleurs, les contemporains de même âge peuvent avoir en commun une certaine "empreinte du temps" (Attias-Donfut, 1988) qui les distingue de leurs aînés comme de leurs cadets, du simple fait qu'ils sont confrontés tout au long de leur vie au même âge à de mêmes états de la société et aux " tendances inhérentes à chaque situation historique " (Mannheim, 1990). Les grands tournants de l'histoire d'une société ou d'un groupe ne transforment pas forcément toutes les cohortes en groupes réels, mais elles marquent durablement leur destinée collective, et peuvent ainsi produire "des dispositions communes [procédant] de la communauté de l'expérience biographique » (Terrail, 1991).

4. En outre, l'embauche à salaire croissant assurait aux fils des revenus dépassant rapidement ceux de leurs pères, qui pouvaient y voir la récompense de leurs investissements éducatifs (Baudelot et Gollac, 1997).

5. On s'en tiendra ici aux évolutions de l'institution scolaire et du marché du travail, qui constituent deux pièces majeures dans la transformation des régimes biographiques. Pour des lectures plus complètes sous l'angle de la succession des générations, voir par exemple (Terrail, 1991) ou (Mauger, 1998). 
place de la famille d'origine dans les débuts de la vie professionnelle sera ensuite abordée à travers l'étude des aides apportées par les parents sur le plan professionnel (6).

\section{Quel devenir pour les enfants d'ouvriers?}

$\mathbf{L}$ a question ouverte "Quelle profession ou quel genre de profession souhaiteriezvous pour votre enfant et pourquoi? » a été posée aux enquêtés ayant un enfant la plupart du temps scolarisé (en primaire, en collège ou au lycée) ou plus rarement un enfant de moins de 18 ans ne suivant plus ou n'ayant jamais suivi d'études (7). Venant à la suite d'une série de questions sur les investissements éducatifs, elle incite donc la personne interrogée à se projeter dans un avenir plus ou moins proche par rapport à la scolarité en cours de l'enfant. Si l'imprécision des réponses au regard du code des catégories socioprofessionnelles (PCS) ne permet pas de juger directement la profession citée en termes de mobilité sociale espérée pour l'enfant (8), une analyse textuelle met à jour des dimensions intéressantes associées à l'avenir professionnel anticipé par les ouvriers pour leurs enfants (9), dans la mesure où une génération se distingue notamment par la façon dont ses membres se représentent le devenir de leurs enfants (cf. encadrés 1 et 2). Peu de professions bien déterminées ressortent des réponses en nombre suffisant pour qu'on puisse les répartir selon des catégories socioprofessionnelles. On se contentera donc tout d'abord d'examiner un palmarès des professions les plus fréquemment citées selon le milieu social des parents (cf. tableau 1).

\section{Les professions de l'enseignement sont parmi les plus citées}

Parmi les plus citées, les professions de l'enseignement semblent constituer des métiers particulièrement attractifs pour les parents ouvriers. Le corps enseignant représente toujours une voie possible et souhaitable de mobilité ascendante pour les enfants d'ouvriers (Terrail, 1984). $9 \%$ des ouvriers souhaitent que leur enfant soit enseignant (du primaire ou du secondaire), contre $6 \%$ des parents, tous milieux

\footnotetext{
6. Ce travail s'appuie essentiellement sur une exploitation secondaire de l'enquête Transmissions familiales, réalisée par I'Insee en 2000 (cf. encadré 1). Les traitements statistiques ont été obtenus à l'aide du logiciel Sas.

7. Dans la population enquêtée ici, la plupart des parents ouvriers (82 \%) sont nés dans les années 1950 et 1960, la grande majorité de leurs enfants (63\%) dans les années $1980.56 \%$ des parents avaient un père ouvrier, $63 \%$ un père né entre 1920 et 1939. Les parents ouvriers enquêtés ici sont donc pour l'essentiel des enfants de cette " génération singulière " décrite par Noiriel (1986) qui, à la suite de l'enracinement ouvrier de leurs parents dans l'entre-deux-guerres, ont connu une ascension au sein du monde ouvrier dans les années 1950 et 1960.

8. En fait, les enquêtés n'ont pas tous répondu de la même manière à la question, certains donnant une désignation plus ou moins précise d'une profession ("gardien de la paix", " infirmière »), d'autres citant plutôt un "genre de profession", c'est-à-dire une activité ou une branche professionnelle sans référence à une position hiérarchique ("commerciale", " mécanique industrielle ») ou encore un statut de travail (" une profession libérale pour qu'il soit tranquille », "fonctionnaire pour la sécurité de l'emploi »).

9. On utilise ici la catégorie socioprofessionnelle de la personne de référence du ménage, sauf pour les citations en exemple où la catégorie socioprofessionnelle de la personne répondante est privilégiée.
}

Tableau 1

Quelques professions souhaitées par les parents pour leurs enfants

Réponse à la question «Quelle profession ou quel genre de profession souhaiteriez-vous pour votre enfant ? "

En \% de réponses

\begin{tabular}{|l|c|c|c|c|c|c|c|c|c|}
\hline $\begin{array}{c}\text { Catégorie } \\
\text { socioprofessionnelle } \\
\text { de la personne de } \\
\text { référence du } \\
\text { ménage }\end{array}$ & $\begin{array}{c}\text { Ne sait } \\
\text { pas }\end{array}$ & $\begin{array}{c}\text { C'est son } \\
\text { choix }\end{array}$ & Enseignant & Médecin & $\begin{array}{c}\text { Dans le } \\
\text { domaine de } \\
\text { l'informatique }\end{array}$ & Ingénieur & Avocat & $\begin{array}{c}\text { Vétérinaire } \\
\text { Profession } \\
\text { artistique }\end{array}$ \\
\hline Ouvriers & 17 & 45 & 9 & 3 & 3 & 1 & 2 & 2 & 1 \\
Autres & 17 & 49 & 4 & 4 & 2 & 2 & 2 & 2 & 3 \\
\hline Ensemble & $\mathbf{1 7}$ & $\mathbf{4 7}$ & $\mathbf{6}$ & $\mathbf{3}$ & $\mathbf{2}$ & $\mathbf{2}$ & $\mathbf{2}$ & $\mathbf{2}$ & $\mathbf{2}$ \\
\hline Test du Chi-deux (1) & $\mathrm{ns}$ & $\mathrm{ns}$ & $* \star *$ & $\mathrm{~ns}$ & $\mathrm{~ns}$ & $*$ & $\mathrm{~ns}$ & $\mathrm{~ns}$ & $*$ \\
\hline
\end{tabular}

1. * signale que le test du Chi-deux comparant les proportions entre les ouvriers et le reste de la population est significatif au seuil de $15 \%,{ }^{\star \star}$ au seuil de $5 \%,{ }^{\star \star \star}$ au seuil de $1 \%$ et ns pour les tests non significatifs.

Lecture : $45 \%$ des parents de milieu ouvrier déclarent que c'est à leur enfant de choisir sa profession (cf. encadré 2).

Champ : individus dont l'enfant est soit scolarisé en primaire, au collège ou au lycée, soit a moins de 18 ans et n'a jamais fait d'études ou n'en suit plus (échantillon de la table des transmissions effectuées) (cf. encadré 1).

Source : enquête Transmissions familiales, Insee, 2000. 
confondus (10). Les raisons avancées tiennent notamment à la recherche de la sûreté de l'emploi («maîtresse pour la sécurité de l'emploi » (réponse du père, un électronicien)) et d'un statut social plus élevé («enseignant pour qu'il soit mieux que nous » (une piqueuse dans la maroquinerie)). Le style de vie associé aux rythmes particuliers du travail des enseignants fait aussi partie des motifs importants cités par les ouvriers qui souhaitent voir leurs enfants s'engager dans ces professions: « institutrice pour avoir la belle vie et plein de vacances » (une soudeuse). Enfin, à côté de la dimension sexuée de ce choix («institutrice pour pouvoir élever ses enfants » (un technicien d'atelier)), le goût de la connaissance et les dispositions scolaires justifient également le choix de ces métiers : «enseignante, elle s'intéresse à tout, elle veut tout connaître » (un grutier).
La profession d'avocat, la médecine et le domaine de l'informatique sont aussi fréquemment cités par les parents de milieu ouvrier que dans les autres milieux. Rarement explicitées, les motivations de ces choix renvoient sans doute avant tout à des désirs de mobilité sociale, comme pour ce maçon souhaitant que son fils soit «médecin pour ouvrir d'autres horizons et connaître ce que nous ne connaissons pas». Aussi souvent que dans d'autres familles, le métier d'avocat est ainsi d'abord envisagé comme une réussite sociale: «avocat, pour réussir sa vie » (un moulinier). Comme dans les autres milieux, le choix du métier de vétérinaire

10. Cette proportion atteint même $11 \%$ chez les ouvriers de père ouvrier. Ont été agrégées pour cet indice les réponses mentionnant les termes suivants : prof, professeur, enseignant, enseignante, maitresse, institutrice, instituteur.

Encadré 1

\section{L'ENQUÊTE TRANSMISSIONS FAMILIALES}

Cette enquête constitue la partie variable de l'Enquête permanente sur les conditions de vie des ménages (EPCVM) réalisée par l'Insee en octobre 2000 auprès de 5299 personnes de 15 ans et plus. Une enquête ponctuelle ne permet pas toujours de démêler les effets propres à l'âge, à la période ou à la génération dans l'analyse des pratiques ou des opinions des enquêtés. Mais le questionnement rétrospectif adopté ici sur des transmissions effectuées à un moment donné de la trajectoire des enfants autorise des comparaisons intergénérationnelles.

\section{Des comparaisons intergénérationnelles}

Les deux caractéristiques principales de l'enquête Transmissions familiales en font, en effet, un matériau particulièrement intéressant pour l'étude de l'évolution de certains rapports sociaux entre les générations. D'une part, elle aborde conjointement plusieurs types de transmissions intergénérationnelles souvent dispersés dans d'autres enquêtes, qu'il s'agisse de transmissions matérielles (héritages, donations et aides financières), de services rendus aux jeunes par leurs parents dans certaines circonstances (accès à l'emploi, au logement) ou encore de transmissions symboliques (investissement éducatif, pratiques culturelles et valeurs). D'autre part, elle permet utilement de comparer les transmissions d'une génération familiale à l'autre, puisqu'elle s'intéresse non seulement à ce qu'ont reçu les enquêtés de leurs parents lors de leur enfance et de leur jeunesse (table des transmissions reçues), mais aussi à ce qu'ils transmettent ou comptent transmettre à leur tour en tant que parents, cette fois vers leurs propres enfants (table des transmissions effectuées), en conservant les mêmes questions le plus souvent dans les deux cas. Si 2038 individus n'ont répondu qu'à la première partie du questionnaire sur les transmissions reçues, les autres ont répondu tantôt au premier module sur les transmissions envers leurs enfants scolarisés (à l'école primaire, au collège ou au lycée) ou de moins de 18 ans ne suivant pas (ou n'ayant jamais suivi) d'études (1 146 répondants), tantôt au second module sur les transmissions envers leurs enfants en études supérieures, ou de plus 18 ans ayant terminé leurs études ou n'en n'ayant jamais suivies (2 115 répondants).

\section{Une analyse textuelle des réponses à une question ouverte}

Pour cet article, on a procédé à une analyse textuelle des réponses à la question ouverte sur le métier souhaité par les parents pour leurs enfants scolarisés (en primaire, en collège ou au lycée) ou, plus rarement, leurs enfants de moins de 18 ans ne suivant plus ou n'ayant jamais suivi d'études : "Quelle profession ou quel genre de profession souhaiteriez-vous pour < prénom de l'enfant > et pourquoi ? » (cf. encadré 2). Dans la partie sur les transmissions reçues, on a également exploité les questions sur l'encouragement reçu des parents à travailler le plus tôt possible ou à continuer les études : "Globalement, vos parents vous ont-ils plutôt poussé à travailler le plus tôt possible? " et (dans le cas d'une réponse négative à cette question) «... à continuer vos études ? ". Enfin, on a examiné les réponses à trois questions sur les aides apportées par les parents à leurs enfants sur le plan professionnel (sans compter les petits boulots et les jobs d'été) : «Sur le plan professionnel, est-il arrivé que vos parents vous fassent entrer dans l'entreprise où ils travaillent?", «... vous aident à trouver du travail par vos relations ? ", «... vous aident financièrement à monter un projet ? ». 
Encadré 2

\section{L'ANALYSE TEXTUELLE DES RÉPONSES À UNE QUESTION OUVERTE}

De plus en plus développée et présente dans beaucoup de logiciels, l'analyse textuelle consiste à compter les occurrences de certaines unités sémantiques dans un texte pour procéder ensuite à des analyses statistiques. On n'a utilisé ici que ses outils les plus simples.

L'analyse textuelle des réponses à la question ouverte sur le métier souhaité par les parents pour leurs enfants a été menée en utilisant dans SAS une macro développée par Olivier Godechot. 7012 mots ont été utilisés par les 1146 répondants à cette question ouverte. On a tout d'abord examiné par fréquence décroissante la liste de ces mots, puis des segments de deux et trois mots (en ne tenant pas compte des mots outils) (cf. tableaux A, B et C). Si l'on s'est intéressé exclusivement aux réponses des parents ouvriers, l'analyse a été menée sur l'ensemble des répondants.

II est vite apparu que les réponses mettant en avant l'autonomie des aspirations de l'enfant avaient une grande importance. C'est pourquoi on a créé une variable à partir de ces mots et segments pour signaler cette référence au choix de l'enfant. De la même façon, on remarque aussi que les réponses révèlent souvent une relative indétermination. Ceci a conduit à créer une variable indiquant que les parents ne savent pas quelle profession ils souhaitent pour leur enfant. Enfin, à partir du palmarès des mots utilisés, on a créé quelques variables regroupant les réponses qui citent une profession ou un genre de profession spécifiques et suffisamment représentées pour les étudier plus particulièrement.

Tableau A

Liste des mots de fréquence supérieure à 9 et de longueur supérieure à deux caractères

\begin{tabular}{|c|c|c|c|c|c|c|c|}
\hline Mot & Fréquence & Mot & Fréquence & Mot & Fréquence & Mot & Fréquence \\
\hline $\mathrm{CHOIX}$ & 89 & CHOISIR & 33 & ENVIE & 20 & DOMAINE & 15 \\
\hline PROFESSION & 76 & TRAVAIL & 31 & PLAISE & 20 & CHOSE & 14 \\
\hline CHOISIRA & 71 & JEUNE & 30 & SOUHAIT & 20 & TRÈS & 14 \\
\hline MÉTIER & 67 & IDÉE & 29 & BON & 19 & AVOCAT & 13 \\
\hline VEUT & 61 & MÉDECIN & 28 & SÉCURITÉ & 19 & RIEN & 13 \\
\hline FAIRE & 57 & PLAÎT & 26 & CELA & 18 & AVOIR & 12 \\
\hline AIME & 47 & ÊTRE & 25 & ENFANTS & 18 & LAISSE & 12 \\
\hline VOUDRA & 42 & LIBRE & 25 & NSP & 18 & AVOCATE & 11 \\
\hline FERA & 41 & SAIS & 25 & PLAIRA & 18 & DÉCIDE & 11 \\
\hline SOUHAITE & 41 & VÉTÉRINAIRE & 25 & INFORMATIQUE & 17 & ENCORE & 11 \\
\hline TROP & 40 & EMPLOI & 22 & INSTITUTRICE & 17 & BONNE & 10 \\
\hline SAIT & 39 & INGÉNIEUR & 22 & ARTISTIQUE & 16 & FONCTIONNAIRE & 10 \\
\hline BIEN & 37 & PROFESSEUR & 22 & DÉCIDERA & 16 & MANUEL & 10 \\
\hline AUCUNE & 34 & MÊME & 21 & FASSE & 16 & VIE & 10 \\
\hline
\end{tabular}

Lecture : les occurrences de ces 56 mots représentent à peu près $22 \%$ de l'ensemble des occurrences des mots utilisés.

Tableau B

Liste des segments de deux mots de fréquence supérieure à 9

\begin{tabular}{|c|c|c|c|c|c|c|c|}
\hline Segments & Fréquence & Segments & Fréquence & Segments & Fréquence & Segments & Fréquence \\
\hline C'EST & 190 & TROP JEUNE & 25 & DE CHOISIR & 18 & IL AIME & 12 \\
\hline QU'ELLE & 137 & AUCUNE IDÉE & 24 & LUI PLAIRA & 18 & IL EST & 12 \\
\hline CE QU' & 136 & CE QUI & 24 & EST CE & 17 & LIBRE CHOIX & 12 \\
\hline QU'IL & 126 & ELLE EST & 24 & IL FERA & 16 & A ENVIE & 11 \\
\hline QUI LUI & 69 & IL VEUT & 24 & JE N' & 16 & ELLE A & 11 \\
\hline SON CHOIX & 57 & SAIS PAS & 24 & ENVIE DE & 15 & ELLE DE & 11 \\
\hline NE SAIT & 38 & DANS LE & 23 & UN TRAVAIL & 15 & IL A & 11 \\
\hline SAIT PAS & 38 & EST A & 23 & L'EMPLOI & 14 & N'EST & 11 \\
\hline PARCE QUE & 37 & LUI QUI & 23 & MÉTIER QUI & 14 & PAS À & 11 \\
\hline EST SON & 36 & EST UN & 22 & PAS D' & 14 & SÉCURITÉ DE & 11 \\
\hline JE NE & 31 & NE SAIS & 22 & UN BON & 14 & VEUT FAIRE & 11 \\
\hline PAS DE & 29 & CAR C' & 21 & A ELLE & 13 & CAR IL & 10 \\
\hline UNE PROFESSION & 28 & ELLE VOUDRA & 21 & AIME LES & 13 & CELLE QUI & 10 \\
\hline ELLE VEUT & 27 & PARCE QU' & 21 & EST PAS & 13 & DANS L' & 10 \\
\hline UN MÉTIER & 27 & QUI CHOISIRA & 21 & N'Al & 13 & DE SON & 10 \\
\hline DE L' & 26 & IL VOUDRA & 20 & POUR LA & 13 & DE SOUHAIT & 10 \\
\hline FERA CE & 26 & LUI PLAISE & 20 & PROFESSION QUI & 13 & ELLE FASSE & 10 \\
\hline LUI PLAIT & 26 & DE FAIRE & 19 & AI PAS & 12 & ELLE MÊME & 10 \\
\hline ELLE CHOISIRA & 25 & ELLE AIME & 19 & BON MÉTIER & 12 & LA PROFESSION & 10 \\
\hline ELLE QUI & 25 & ELLE FERA & 19 & CAR ELLE & 12 & LE MÉTIER & 10 \\
\hline EST ELLE & 25 & IL CHOISIRA & 19 & CELLE QU' & 12 & & \\
\hline EST LUI & 25 & QUE C' & 19 & EST TROP & 12 & & \\
\hline
\end{tabular}

Lecture : les occurrences de ces 86 segments représentent à peu près $37 \%$ de l'ensemble des occurrences de segments de deux mots utilisés. 
est davantage celui de l'enfant : «vétérinaire car elle est attirée par les animaux » (un menuisier). En revanche, les professions artistiques ou les métiers d'ingénieur sont légèrement moins présents que dans les réponses des familles non ouvrières.

\section{Respecter avant tout le choix de l'enfant}

Mais est-il sûr que tous les parents aient un avis sur la question? Les ouvriers ne se distinguent pas significativement des autres parents à cet égard : $17 \%$ d'entre eux expriment la même indétermination lors de l'enquête (cf. tableau 1). L'absence de souhait professionnel pour leur enfant y apparaît à travers une variante des réponses suivantes: «je ne sais pas », «pas d'opinion», «aucune», "illelle est trop jeune », "pas d'a priori », «n'importe », etc. Il peut s'agir alors de préserver l'enfant d'un choix professionnel précoce : «elle est trop jeune pour y penser» (un scieur de pierre). Parfois le parcours scolaire difficile semble interdire toute projection dans un futur professionnel, comme dans ces réponses : «je ne sais pas, avec son niveau très bas » (un boucher), " pas la peine de chercher, on ne sait pas ce qu'on va en faire » (un préparateur de commandes).

Il est également tentant de discerner dans ce brouillage d'un avenir trop lointain une conséquence de l'allongement inédit des scolarités dans les lignages ouvriers, empêchant la formulation d'aspirations bien définies par les parents. Mais ce qui frappe avant tout dans leurs répon- ses, c'est plutôt le sentiment qu'il n'est pas de leur responsabilité de désigner un métier à leurs enfants. En permettant l'entrée des ouvriers dans le modèle des études longues, le dispositif de l'école unique mis en place par les réformes scolaires de 1959 à 1975 a fait des trajectoires scolaires et professionnelles l'affaire privée des jeunes eux-mêmes. Effectivement, les parents hésitent souvent à répondre parce qu'ils semblent donner la priorité aux choix professionnels de l'enfant lui-même. Qu'ils formulent tout de même un souhait professionnel ou non, $47 \%$ des parents invoquent, en effet, dans leur réponse la primauté du désir de l'enfant dans le choix de sa profession (cf. tableau 1) (11). Sans doute cette tendance à valoriser l'autonomie des décisions des enfants est-elle encore plus répandue parmi les cadres ou les professions intermédiaires (elle apparaît respectivement dans $54 \%$ et $52 \%$ de leurs réponses), mais il est plus remarquable encore qu'elle soit également très significative chez les ouvriers (45\% des réponses) car elle n'allait sans doute pas de soi dans les générations ouvrières les plus anciennes : la volonté de l'enfant n'apparaît que dans $31 \%$ des réponses des ouvriers nés entre 1938 et 1952 contre $48 \%$ dans les cohortes d'ouvriers nés entre 1953 et 1967 (cf. tableau 2). Cette progression du libre choix laissé à l'enfant d'une génération à l'autre n'est d'ailleurs statistiquement significative que pour les ouvriers.

11. Proportion obtenue en regroupant toutes les réponses où apparaissent les expressions suivantes : " c'est à elle / lui de ... ", "c'est pas à moi de... ", " ça sera son choix ", " ce qu'elle / il souhaite / décide ", « ce qui lui conviendra / plaira / plait / lui fera plaisir », « elle / il est libre », etc.

Encadré 2 (suite)

Tableau C

Liste des segments de trois mots de fréquence supérieure à 9

\begin{tabular}{|l|c|l|c|l|c|l|c|}
\hline \multicolumn{1}{|c|}{ Segments } & Fréquence & \multicolumn{1}{|c|}{ Segments } & Fréquence & \multicolumn{1}{|c|}{ Segments } & Fréquence & \multicolumn{1}{|c|}{ Segments } & Fréquence \\
\hline CE QU'ELLE & 69 & QU'IL VEUT & 23 & JE NE SAIS & 17 & EST A ELLE & 11 \\
CE QU'IL & 67 & C'EST A & 22 & EST CE QU' & 15 & N'AI PAS & 11 \\
NE SAIT PAS & 38 & NE SAIS PAS & 22 & ELLE QUI CHOISIRA & 14 & N'EST PAS & 11 \\
C EST SON & 35 & C'EST UN & 21 & PARCE QU'ELLE & 14 & SÉCURITÉ DE L' & 11 \\
EST SON CHOIX & 26 & CAR C'EST & 21 & QUI LUI PLAIRA & 14 & UN MÉTIER QUI & 11 \\
C'EST ELLE & 25 & QU'ELLE VOUDRA & 20 & DE L'EMPLOI & 13 & PAS DE SOUHAIT & 10 \\
C'EST LUI & 25 & QU'IL VOUDRA & 20 & ENVIE DE FAIRE & 13 & PROFESSION QUI LUI & 10 \\
EST ELLE QUI & 25 & PARCE QUE C' & 19 & IL FERA CE & 13 & QU'IL AIME & 10 \\
FERA CE QU' & 24 & QUE C'EST & 18 & JE N'Al & 13 & UN BON MÉTIER & 10 \\
CE QUI LUI & 23 & QUI LUI PLAISE & 18 & QU'ELLE AIME & 13 & & \\
EST LUI QUI & 23 & QUI LUI PLAIIT & 18 & Ȧ ELLE DE & 11 & & \\
QU'ELLE VEUT & 23 & C'EST CE & 17 & ELLE FERA CE & 11 & & \\
\hline
\end{tabular}

Lecture : les occurrences de ces 45 segments représentent à peu près $19 \%$ de l'ensemble des occurrences de segments de trois mots utilisés. 
Beaucoup de parents ouvriers se déclarent ainsi attachés à ce principe d'autodétermination des jeunes: «aucune idée, je la laisserai libre de son choix » (un manutentionnaire), «c'est eux qui décident » (une couturière), «ça, c'est son choix» (un monteur-soudeur), "elle fera son choix, je n'ai pas à l'emmener sur une voie ou une autre » (un magasinier), "Irène a déjà son idée, je n'ai pas à intervenir dans sa décision » (une femme de ménage). Certains parents semblent également épouser le souhait professionnel de leurs enfants : "puéricultrice, car c'est ça qu'elle veut faire» (une ouvrière marầchère), "elle veut s'occuper de gestion et d'administration, on souhaite qu'elle y arrive » (un chauffeur). Si le libre choix de l'enfant est ainsi mis en avant, c'est aussi parce que les parents leur souhaitent un travail qui leur apporte un enrichissement personnel : «ce qui lui plaira, parce qu'il vaut mieux faire un boulot $q u$ 'on aime» (un agenceur), «la profession qu'elle voudra pour son épanouissement » (un cariste).

Si l'avenir professionnel souhaité peut paraître ainsi relativement indéterminé et laissé au libre choix de l'enfant, il est notable en revanche que les trajectoires ouvrières soient quasi absentes des réponses. L'aspiration à l'autonomie et à la réalisation de soi s'accommode mal des emplois salariés d'exécution. Des termes comme « usine », « manuel » ou « ouvrier » sont très rarement utilisés par les enquêtés, et presque toujours de manière négative, comme des repoussoirs. Il s'agit alors d'un destin rejeté pour les enfants (comme pour ce conducteur d'installations automatisées : "j'espère seulement qu'elle ne travaillera pas en usine comme nous »), ou d'une éventualité envisagée en cas de difficultés scolaires (un mécanicien souhaite ainsi que son fils soit «travailleur manuel, [car] il n'aime pas trop l'école »). Seuls deux enquêtés ouvriers citent positivement le travail manuel comme souhait pour leurs enfants: « qu'il apprenne un bon métier manuel, car on manquera toujours d'ouvriers spécialisés » (un ouvrier d'usine), "coiffeuse, c'est un travail manuel, on a toujours besoin d'un coiffeur» (un chauffeur de bus).

\section{La réussite scolaire : un sésame pour l'emploi}

La destinée des enfants n'est toutefois pas uniquement subordonnée à leurs propres choix et désirs. Dans beaucoup de réponses, il est clair qu'il ne suffit pas de vouloir pour pouvoir et les souhaits professionnels sont conditionnés par la scolarité. La longueur et la qualité du parcours scolaire, qui constituent bien souvent un sésame pour l'emploi et la carrière, sont évoquées comme préalables au libre choix de la profession : «elle sera libre, mais il faut qu'elle fasse des études » (un jardinier), «cela dépend de son niveau d'études, il choisira» (un régleur-ajusteur), "ce qu'elle choisira, elle a des facilités, et elle encore jeune pour savoir» (un chauffeur), "qu'elle aille d'abord le plus loin possible à l'école, elle veut être avocate» (un carreleur). Ces références aux études des enfants dans les réponses sur le choix du métier montrent bien la préoccupation scolaire grandissante dans les familles ouvrières.

Toutes choses égales par ailleurs, plus les parents ont prolongé leurs études, plus ils ont tendance à mettre en avant l'autonomie des

Tableau 2

Libre choix de la profession par l'enfant selon les cohortes et le milieu social

Réponse à la question : "Quelle profession ou quel genre de profession souhaiteriez-vous pour votre enfant ? »

En \% de réponses indiquant que c'est à l'enfant de choisir sa profession

\begin{tabular}{|c|c|c|c|}
\hline \multirow{2}{*}{$\begin{array}{c}\text { Catégorie socioprofessionnelle } \\
\text { de la personne de référence } \\
\text { du ménage }\end{array}$} & \multicolumn{2}{|c|}{ L'enquêté est né entre... } & \multirow{2}{*}{ Ensemble } \\
\hline & $\ldots 1938$ et 1952 & ... 1953 et 1967 & \\
\hline Indépendants & ns & 38 & 44 \\
\hline Cadres et professions intermédiaires & 58 & 53 & 54 \\
\hline Employés & ns & 46 & 46 \\
\hline Ouvriers (1) & $31^{*}$ & $48^{*}$ & 44 \\
\hline Ensemble & 47 & 48 & 48 \\
\hline
\end{tabular}

Lecture : la variable indiquant que c'est à l'enfant de choisir sa profession a été construite à partir de l'analyse textuelle d'une question ouverte (cf. encadré 2). Lorsque les effectifs sont trop fragiles pour calculer des pourcentages, on l'a signalé par ns.

Champ : individus actifs nés entre 1938 et 1967 dont l'enfant est soit scolarisé en primaire, au collège ou au lycée, soit a moins de 18 ans et n'a jamais fait d'études ou n'en suit plus (échantillon de la table des transmissions effectuées) (cf. encadré 1).

Source : enquête Transmissions familiales, Insee, 2000. 
enfants dans le choix de leur profession. Tout en restant majoritaire, cette propension décline quand ils estiment que leur enfant connaît des difficultés, voire de grosses difficultés dans sa scolarité. C'est ce qui ressort d'un modèle logistique contrôlant également les effets du milieu social, du sexe de l'enfant et de la cohorte de naissance des enquêtés (cf. graphique I). Si ces dernières dimensions ne semblent pas exercer d'influence significative sur le taux de réponses donnant la priorité aux choix professionnels de l'enfant, l'histoire scolaire des parents et des enfants l'influencent en revanche sensiblement. Tout se passe comme si la réussite scolaire encourageait les parents à laisser leurs enfants déterminer eux-mêmes leur avenir dans le champ des possibles professionnels, chez les ouvriers comme dans les autres groupes sociaux.

\section{Des aspirations aux études longues de plus en plus fortes}

Espoir de mobilité sociale, relative indétermination du futur des enfants, rejet du travail ouvrier, aspiration à l'épanouissement personnel au travail, ambitions scolaires: toutes ces dispositions ne sont pas partagées également, ni au même degré, ni de la même façon par tous les ouvriers de cette génération nés dans les années 1950 et 1960 . Ces dispositions les distinguent cependant de la génération ouvrière qui les précède, en particulier par la place nouvelle qu'y occupe la préoccupation scolaire. Quels que soient les horizons sociaux envisagés, l'institution scolaire est en effet devenue, en quelques décennies, un point de passage obligé et apparaît même comme la principale matrice des destinées individuelles aux yeux de bien des parents. Qu'il s'agisse de gravir des échelons dans les rangs des qualifications ouvrières, de quitter la condition ouvrière pour se mettre à son compte (comme artisan ou commerçant) ou encore prendre place dans le salariat d'encadrement, les destins sociaux des enfants d'ouvriers peuvent de moins en moins faire l'économie du diplôme. Minoritaires il y a encore une quarantaine d'années, les aspirations ouvrières aux études longues sont non seulement devenues majoritaires aujourd'hui mais l'idée s'est de plus en plus répandue dans les familles que les

\section{Graphique I \\ Modélisation de la modalité de réponse : “C'est à l'enfant de choisir sa profession »}

Situation de référence en italique

CSP de la personne de référence du ménage

Agriculteur ou indépendant

Employé

Ouvrier

Cadre ou profession intermédiaire

Sexe de l'enfant

Femme

Homme

Niveau scolaire de l'enfant dans l'ensemble pendant sa scolarité

Un bon ou un excellent élève

Un élève moyen

Un élève qui a des difficultés ou des grosses difficultés

Diplôme de l'enquêté

Inférieur ou égal au CEP

CAP, BEP ou BEPC

Supérieur ou égal au bac

Cohorte de naissance de l'enquêté

1945-1959

1960-1974

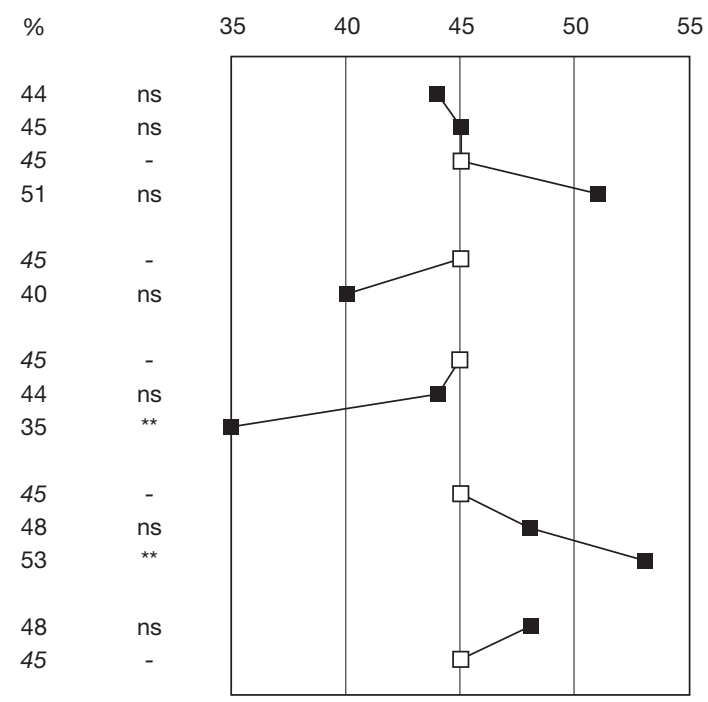

Lecture : la modalité qu'on cherche à expliquer (indiquant que les parents laissent aux enfants le choix de leur profession) est issue du recodage des réponses à la question ouverte sur la profession souhaitée pour les enfants (cf. encadré 2). II s'agit d'un modèle de type logit. On part d'une situation de référence signalée en italique : un enquêté né entre 1960 et 1974, diplômé au mieux du CEP, dont la personne de référence du ménage est ouvrière et ayant une fille bonne ou excellente élève a $45 \%$ de chances de déclarer que « c'est à l'enfant de choisir sa profession ". Le modèle permet d'évaluer que dans une famille où l'enfant a des difficultés scolaires et présentant par ailleurs toutes les caractéristiques de cette situation de référence, l'enquêté aurait $35 \%$ de chances de répondre de la même façon. L'écart des chances (10 points) mesure ainsi l'effet propre des performances scolaires de l'enfant sur la propension des parents à laisser l'enfant autonome dans le choix de sa profession. Dans la seconde colonne, ${ }^{* *}$ indique que le paramètre estimé est significatif au seuil de $5 \%$ et ns pour un test non significatif.

Champ : enquêtés nés entre 1945 et 1974, ayant un enfant scolarisé en primaire, en collège ou au lycée ou un enfant de moins de 18 ans qui a terminé ses études ou n'en a pas fait (échantillon de la table des transmissions effectuées, $N=1098$ ). Les non-réponses ont été enlevées de l'échantillon pour la régression logistique.

Source : enquête Transmissions familiales, Insee, 2000. 
enfants doivent «aller le plus loin possible à l'école ».

Le déclin de l'incitation parentale à travailler le plus tôt possible de génération en génération témoigne ainsi de l'évolution de la politique éducative des familles face au rôle nouveau du diplôme dans le modelage des trajectoires sociales (cf. tableau 3) (12). Encore importante pour les enfants d'ouvriers nés entre les deux guerres, voire jusqu'au milieu des années 1950, elle baisse fortement ensuite pour ne plus concerner, dans les cohortes les plus récentes, qu'une petite minorité, comme pour les enfants des autres catégories sociales. Les enfants d'ouvriers sont au contraire de plus en plus fréquemment poussés à continuer leurs études. En la matière, les différences de comportement avec les familles de cadres supérieurs ou de professions libérales se sont beaucoup estompées. L'essor actuel de l'encouragement à poursuivre des études dans les milieux ouvriers est spectaculaire, notamment chez les garçons (13), pour les générations arrivant au collège entre le début des années 1980 et le milieu des années 1990, au moment précisément de la mise en place des bacs profes- sionnels censés conduire $80 \%$ d'une classe d'âge au niveau du bac (Beaud, 2002).

\section{L'ambition scolaire se généralise}

Plusieurs facteurs influent simultanément sur la propension des parents à inciter leurs enfants à travailler le plus tôt possible ou, à l'inverse, à continuer leurs études : la cohorte de naissance des enfants en est un, le sexe de l'enfant en est un autre. Le passé scolaire des parents, ainsi que leur milieu social, entrent aussi en ligne de compte. Si l'on cherche à hiérarchiser les effets de chacune de ces variables, on peut procéder à des analyses statistiques toutes choses égales par ailleurs, prenant en considération toutes ces

12. L'origine sociale est ici repérée par la profession du père pendant la scolarité de l'enquêté.

13. Si les filles d'ouvriers ont ainsi été davantage encouragées à continuer leurs études que les fils d'ouvriers dans les années 1960 et 1970 (Terrail, 1992), le rattrapage de l'encouragement des garçons est ici remarquable dans les années 1980 et 1990, lors de la seconde explosion scolaire.

Tableau 3

Attitudes des parents par rapport à la prolongation des études

A - Réponse à la question : “ Vos parents vous ont-ils poussé à travailler le plus tôt possible ?»

En \% de réponses positives selon la profession du père de l'enquêté

\begin{tabular}{|l|c|c|}
\hline \multicolumn{1}{|c|}{ Cohorte de naissance } & Ensemble & Père ouvrier \\
\hline De 1910 à 1924 & 43 & 54 \\
De 1925 à 1939 & 41 & 44 \\
De 1940 à 1954 & 34 & 35 \\
De 1955 à 1969 & 19 & 22 \\
De 1970 à 1984 & 13 & 14 \\
\hline
\end{tabular}

Lecture : 54 \% des enquêtés nés de père ouvrier entre 1910 et 1924 ont été poussés à travailler le plus tôt possible. Champ : personnes de plus de 15 ans suivant ou ayant suivi des études, élevées par au moins un des parents (échantillon de la table des transmissions reçues) (cf. encadré 1).

B - Réponse à la question : « Vos parents vous ont-ils poussé à continuer vos études ? »

En \% de réponses positives selon le sexe de l'enquêté et la profession de son père

\begin{tabular}{|l|c|c|c|c|c|c|}
\hline \multicolumn{1}{|c|}{ Cohorte de naissance } & Ensemble & Hommes & Femmes & Enfant d'ouvrier & Fils d'ouvrier & Fille d'ouvrier \\
\hline De 1910 à 1924 & 36 & $\mathrm{~ns}$ & $\mathrm{~ns}$ & 36 & $\mathrm{~ns}$ & $\mathrm{~ns}$ \\
De 1925 à 1939 & 44 & 47 & 41 & 30 & 34 & 27 \\
De 1940 à 1954 & 61 & 62 & 58 & 55 & 55 & 56 \\
De 1955 à 1969 & 64 & 62 & 66 & 56 & 52 & 60 \\
De 1970 à 1984 & 81 & 82 & 81 & 78 & 82 & 74 \\
\hline
\end{tabular}

Lecture : $82 \%$ des fils d'ouvriers nés entre 1970 et 1984 ont été poussés à continuer leurs études. On a indiqué par ns les cases où les effectifs sont trop fragiles pour calculer des pourcentages.

Champ : personnes de plus de 15 ans suivant ou ayant suivi des études, élevées par au moins un des parents, n'ayant pas été poussées par leurs parents à travailler le plus tôt possible (échantillon de la table des transmissions reçues) (cf. encadré 1).

Source : enquête Transmissions familiales, Insee, 2000 
variables simultanément (cf. graphique II) (14). Le groupe socioprofessionnel du père est ainsi une dimension dont l'effet demeure déterminant sur l'incitation faite aux enfants à prolonger leurs études. Mais si l'on retrouve ici l'écart d'ambition scolaire selon l'origine sociale déjà mis en évidence par les chercheurs de l'Ined au début des années 1960 (Ined, 1970), on notera aussi que dans tous les milieux (à part chez les agriculteurs), les parents souhaitent aujourd'hui très majoritairement que les enfants poursuivent leurs études. L'ampleur du différentiel d'ambition scolaire manifesté par les parents selon leur milieu social est ici minime, ce qui affaiblit la thèse de Boudon sur les inégalités scolaires (15).

Mais il faut tenir compte également, à milieu comparable, des parcours scolaires des parents.
Là aussi, l'incitation à prolonger les études est majoritaire, quel que soit le niveau d'études des parents. De manière générale, plus les parents ont prolongé leurs études, plus ils poussent leurs enfants à le faire également. Enfin, au fil des générations, les parents souhaitent de plus en plus que leurs enfants continuent à étudier. L'essor de la préoccupation scolaire est bien un phénomène générationnel, qui renvoie notamment au déclin de la valeur (d'usage et marchande) de la force de travail simple entre les

14. On a utilisé pour cette analyse une régression logistique (cf. note de lecture du graphique I).

15. Selon Boudon (1973), comparant les coûts, les rendements et les risques escomptés de la prolongation de la scolarité (trois dimensions déterminées par leur position sociale), les ouvriers renoncent précocement à conduire leurs enfants le plus loin possible dans les études.

Graphique II

Situation de référence en italique

CSP du père de l'enquêté

Agriculteur

Ouvrier

Indépendant

Employé

Cadre ou profession intermédiaire

CSP de la mère de l'enquêté

Ouvrière

Employée

Inactive

Cadre ou profession intermédiaire

Agricultrice

Indépendante

Sexe de l'enquêté

Femme

Homme

Niveau d'étude de la mère de l'enquêté

N'a pas été plus loin que la dernière année de primaire

A préparé un diplôme de type CAP ou BEP

Enseignement technique ou professionnel long, enseignement supérieur

$1^{\mathrm{er}}$ ou second cycle d'enseignement général, EPS, BS

Niveau d'étude du père de l'enquêté

N'a pas été plus loin que la dernière année de primaire

A préparé un diplôme de type CAP ou BEP

$1^{\text {er }}$ ou second cycle d'enseignement général, EPS, BS

Enseignement technique ou professionnel long, enseignement supérieur

Cohorte de naissance de l'enquêté

1925-1944

1945-1964

1965-1984

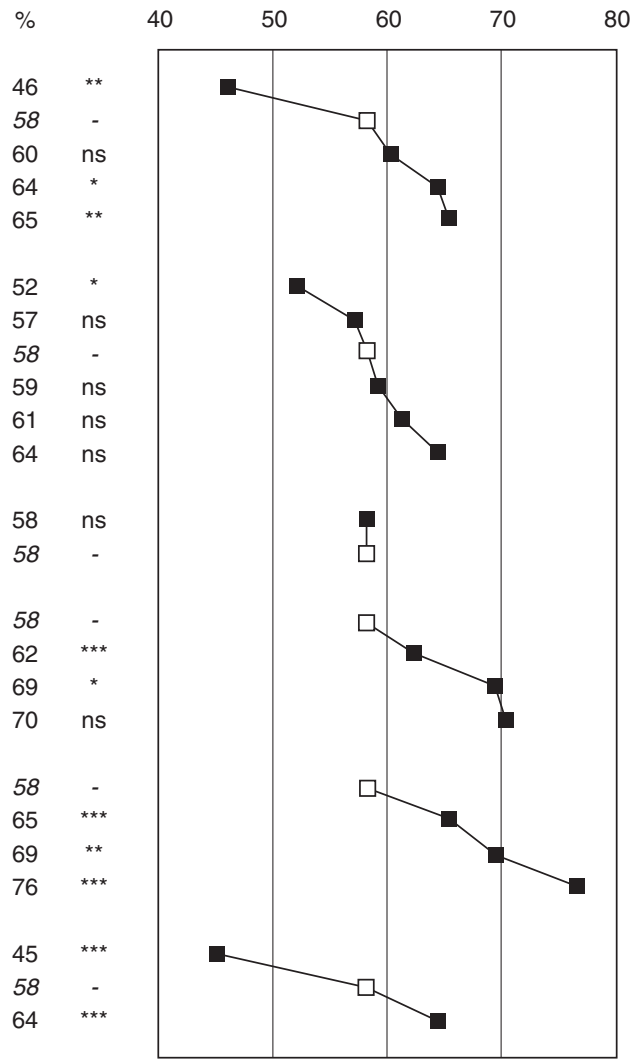

Lecture : il s'agit d'un modèle de type logit. On part d'une situation de référence signalée en italique : un homme né entre 1945 et 1964 de père ouvrier et de mère inactive n'ayant tous deux pas dépassé la dernière année de primaire a $58 \%$ de chances d'avoir été poussé par ses parents à continuer ses études. Le modèle permet d'évaluer qu'une personne née entre 1925 et 1944 et présentant par ailleurs toutes les caractéristiques de cette situation de référence aurait $45 \%$ de chances de répondre de la même manière. L'écart des chances (13 points) mesure ainsi l'effet propre de la cohorte de naissance sur l'incitation parentale à prolonger les études. Dans la seconde colonne, ** indique que le paramètre estimé est significatif au seuil de $5 \%{ }^{* * *}$ pour un seuil de $1 \%$, * pour un seuil de $10 \%$, ns pour un test non significatif).

Champ : individus nés entre 1925 et 1984, élevés par leurs parents, ayant suivi des études et n'ayant pas été poussés par leurs parents à travailler le plus tôt possible. Les non-réponses ont été enlevées de l'échantillon pour la régression logistique (échantillon de la table des transmissions reçues, $N=2938$ )

Source : enquête Transmissions familiales, Insee, 2000 
années 1960 et aujourd'hui, mais aussi conjointement à l'intériorisation chez les parents du rôle de plus en plus déterminant des diplômes les plus élevés dans les recrutements professionnels.

\section{Les parents reportent leurs aspirations sur leurs enfants}

La majorité des ouvriers pensent aujourd'hui que leur enfant aurait dû pousser plus loin ses études. Le bac semble bien être l'objectif minimal : lorsque l'enfant a poursuivi des études au-delà du bac, plus de la moitié des parents ouvriers regrettent encore qu'il n'ait pas prolongé plus loin ses études (cf. tableau 4). La norme du prolongement des études semble bien en passe de se généraliser dans les familles ouvrières. Mais cette règle ne s'impose pas complètement de l'extérieur aux familles, elle est portée par l'expérience biographique des parents. Plus ils ont eux-mêmes prolongé leurs études, plus ils trouvent trop précoce l'arrêt des études de leur enfant. Quel que soit leur diplôme et celui de leur enfant, ils regrettent d'autant plus la fin prématurée des études de leur enfant qu'eux-mêmes ont le sentiment d'avoir interrompu trop tôt les leurs. Ceux qui sont les plus nombreux à trouver que leur enfant aurait dû pousser plus loin ses études sont ainsi les parents qui ont obtenu un diplôme supérieur au certificat d'études, a fortiori quand leurs enfants n'ont pas dépassé le bac. Le regret d'avoir dû interrompre trop tôt ses études est aussi toujours plus marqué chez les femmes en milieu ouvrier, ce qui est sans doute en rapport avec les progrès de la scolarisation et de l'activité féminine dans les dernières décennies (cf. tableau 5).

Après les premiers pas de leurs enfants sur le marché du travail, les parents estiment donc, souvent a posteriori, qu'ils auraient dû poursuivre plus longtemps leurs études et ce, d'autant plus souvent qu'ils ont eux-mêmes éprouvé le sentiment d'avoir interrompu trop tôt les leurs. Quand on cherche à évaluer le rôle de cette frustration des parents sur l'expression des regrets que leurs enfants aient arrêté eux aussi trop tôt leurs études, tout en contrôlant d'autres paramètres pouvant également l'influencer, le report des aspirations scolaires d'une génération à

Tableau 4

Aspirations scolaires pour les enfants en milieu ouvrier selon le sentiment des parents par rapport à leur propre scolarisation

Réponse à la question : "Pensez-vous que votre enfant aurait dû pousser plus loin ses études ? »

En \% de réponses positives

\begin{tabular}{|c|c|c|c|c|}
\hline Diplôme le plus élevé obtenu par l'enfant $\rightarrow$ & & \multirow{2}{*}{ Inférieur au bac } & \multirow{2}{*}{ Bac et plus } & \multirow{2}{*}{ Ensemble } \\
\hline$\downarrow$ Diplôme le plus élevé obtenu par l'enquêté & & & & \\
\hline \multirow{4}{*}{ Sans diplôme } & \multicolumn{4}{|c|}{ Avez-vous le sentiment d'avoir interrompu trop tôt vos études? } \\
\hline & Oui & 52 & 69 & 56 \\
\hline & Non & 52 & 42 & 51 \\
\hline & Ensemble & 52 & 58 & 53 \\
\hline \multirow{4}{*}{ CEP, DFEO (diplôme de fin d'études obligatoires) } & \multicolumn{4}{|c|}{ Avez-vous le sentiment d'avoir interrompu trop tôt vos études? } \\
\hline & Oui & 75 & 56 & 67 \\
\hline & Non & 52 & 43 & 48 \\
\hline & Ensemble & 63 & 49 & 58 \\
\hline \multirow{4}{*}{ BEPC, CAP, BEP, Bac et plus } & \multicolumn{4}{|c|}{ Avez-vous le sentiment d'avoir interrompu trop tôt vos études? } \\
\hline & Oui & 79 & 71 & 76 \\
\hline & Non & 59 & 60 & 59 \\
\hline & Ensemble & 67 & 63 & 65 \\
\hline \multirow{4}{*}{ Ensemble } & \multicolumn{4}{|c|}{ Avez-vous le sentiment d'avoir interrompu trop tôt vos études ? } \\
\hline & Oui & 64 & 64 & 64 \\
\hline & Non & 54 & 50 & 52 \\
\hline & Ensemble & 58 & 56 & 58 \\
\hline
\end{tabular}

Lecture : 67 \% des ouvriers ayant obtenu un diplôme supérieur ou égal au BEPC et ayant un enfant non bachelier pensent que cet enfant aurait dû prolonger ses études. Cette proportion passe à 79 \% lorsqu'ils ont le sentiment d'avoir eux-mêmes interrompu trop tôt leurs études.

Champ : personnes appartenant à un ménage ouvrier ayant un enfant ayant terminé ses études (échantillon de la table des transmissions effectuées) (cf. encadré 1).

Source : enquête Transmissions familiales, Insee, 2000. 
l'autre apparaît nettement (cf. graphique III). Les regrets des parents sont également d'autant plus fréquents que l'enfant a poussé moins loin ses études. Mais quel que soit le diplôme obtenu par ce dernier, plus de la moitié d'entre eux auraient préféré qu'il prolonge son parcours scolaire. On retrouve par ailleurs un effet de génération: avec le temps, la prise de cons-

Tableau 5

Sentiments des enfants d'ouvriers par rapport à la prolongation de leurs études

Réponse à la question : "Avez-vous le sentiment d'avoir dû interrompre trop tôt vos études ? "

En \% de réponses positives selon le sexe et la cohorte de naissance de l'enquêté

\begin{tabular}{|c|c|c|c|c|}
\hline Cohorte de naissance & Profession du père & Hommes & Femmes & Ensemble \\
\hline \multirow{3}{*}{ De 1921 à 1935} & Ouvrier & 50 & 54 & 53 \\
\hline & Autre & 44 & 50 & 48 \\
\hline & Ensemble & 46 & 51 & 49 \\
\hline \multirow{3}{*}{ De 1936 à 1950} & Ouvrier & 39 & 48 & 44 \\
\hline & Autre & 36 & 46 & 42 \\
\hline & Ensemble & 37 & 47 & 42 \\
\hline \multirow{3}{*}{ De 1951 à 1965} & Ouvrier & 41 & 53 & 48 \\
\hline & Autre & 30 & 39 & 35 \\
\hline & Ensemble & 34 & 45 & 40 \\
\hline \multirow{3}{*}{ De 1966 à 1980} & Ouvrier & 30 & 45 & 39 \\
\hline & Autre & 28 & 30 & 29 \\
\hline & Ensemble & 29 & 36 & 33 \\
\hline
\end{tabular}

Lecture : $46 \%$ des hommes nés entre 1921 et 1935 ont le sentiment d'avoir interrompu trop tôt leurs études. Cette proportion passe à $50 \%$ quand leur père était ouvrier.

Champ : personnes de plus de 15 ans ayant terminé leurs études, nées entre 1921 et 1980.

Source : enquête Transmissions familiales, Insee, 2000.

Graphique III

Modélisation de la modalité de réponse : « Les parents pensent que leur enfant aurait dû pousser plus loin ses études”

Situation de référence en italique

CSP de l'enquêté

Employé

Cadre ou profession intermédiaire

Ouvrier

Indépendant ou agriculteur

Diplôme de l'enquêté

CAP ou BEP

Inférieur ou égal au BEPC

Supérieur ou égal au bac

Sentiment de l'enquêté par rapport à l'interruption de ses études

Sentiment d'avoir interrompu trop tôt ses études

Pas le sentiment d'avoir interrompu trop tôt ses études

Cohorte de naissance de l'enfant de l'enquêté

1971-1985

1956-1970

1941-1955

Diplôme de l'enfant de l'enquêté

CAP ou BEP

Supérieur ou égal au bac

Inférieur ou égal au BEPC

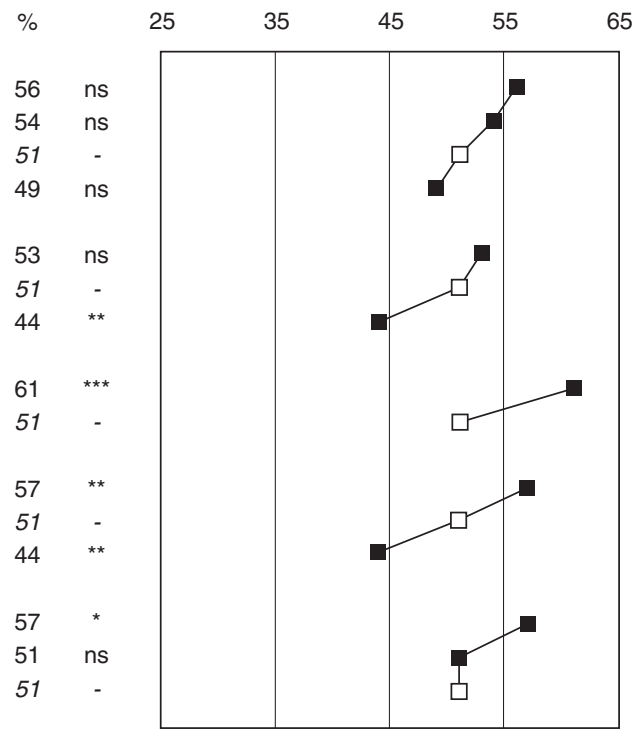

Lecture : il s'agit d'un modèle de type logit. On part d'une situation de référence signalée en italique : un enquêté appartenant à un ménage ouvrier ne possédant pas de diplôme supérieur au BEPC et n'ayant pas le sentiment d'avoir interrompu trop tôt ses études, dont l'enfant né entre 1956 et 1970 et n'a pas obtenu non plus de diplôme supérieur au BEPC a 51 \% de chances de penser que cet enfant aurait dû pousser plus loin ses études. Le modèle permet d'évaluer qu'un enquêté ayant le sentiment d'avoir interrompu trop tôt ses études et présentant par ailleurs toutes les autres caractéristiques de cette situation de référence aurait $61 \%$ de chances de répondre de la même manière. L'écart des chances (10 points) mesure ainsi l'effet propre de la frustration des parents par rapport à l'interruption précoce de leurs propres études sur leurs regrets que l'enfant n'ait pas continué ses études. Dans la seconde colonne, ${ }^{*}$ indique que le paramètre estimé est significatif au seuil de $5 \%\left(^{* * *}\right.$ pour un seuil de $1 \%$, ns pour un test non significatif).

Champ : enquêtés dont l'enfant est né entre 1941 et 1985, a été élevé par ses deux parents, a suivi des études et les a terminées (échantillon de la table des transmissions effectuées, $N=1666$ ). Les non-réponses ont été enlevées de l'échantillon pour la régression logistique. Source : enquête Transmissions familiales, Insee, 2000. 
cience de l'importance des diplômes dans la destinée sociale des enfants est de plus en plus massive. Le diplôme détenu par les parents module sensiblement leur regret que les enfants n'aient pas poursuivi leurs études : les moins diplômés d'entre eux sont aussi ceux qui regrettent le plus que leurs enfants n'aient pas continué plus loin. Les familles ouvrières ne se distinguent cependant pas significativement, dans ce modèle, des familles d'autres milieux.

\section{Un enjeu incontournable pour l'avenir de la lignée}

Cet essor de la préoccupation scolaire s'alimente donc dans l'histoire de la famille et varie selon les performances scolaires des enfants. Il se traduit, d'ailleurs, dans la vie familiale par des discussions de plus en fréquentes et parfois conflictuelles autour de l'école, ce qui atteste bien l'enjeu désormais incontournable que constitue la scolarité pour l'avenir de la lignée.

Des cohortes nées entre 1916 et 1925 aux cohortes nées entre 1966 et 1975, la part des ouvriers qui discutaient de l'école avec leurs enfants est passée de $60 \%$ à $85 \%$. La question scolaire occupe bien aujourd'hui une place centrale dans la vie familiale, au point que lorsqu'il y a des conflits entre parents et enfants, ils se nouent le plus souvent autour de la scolarité. Des cohortes nées entre 1916 et 1935 à celles nées entre 1956 et 1975 , la part des enquêtés qui citent un conflit grave avec leurs parents à propos de l'école ou des études double de $19 \%$ à $38 \%$ parmi ceux qui ont connu au moins un conflit. Pour ceux dont le père était ouvrier, elle passe de $16 \%$ à $42 \%$. C'est de loin la première source de conflit avec les enfants citée par les parents ayant des enfants encore scolarisés ou de moins de 18 ans non scolarisés (nés entre 1976 et 1995) : $56 \%$ des enquêtés ayant connu au moins un conflit avec leur enfant reconnaissent qu'il leur est arrivé d'être en conflit grave avec leur enfant à propos de l'école ou de ses études. On trouve sensiblement la même proportion (53\%) dans les familles ouvrières (16).

\section{Une appropriation des enjeux scolaires au fil des générations}

L'emprise croissante et généralisée de la socialisation scolaire sur les biographies dans les catégories ouvrières contribue ainsi fortement à transformer le mode de constitution des générations. Non seulement parce qu'un modèle de prolongation maximale des cursus scolaires dans les catégories qui en étaient le plus éloignées auparavant tend à s'imposer, mais aussi parce que ce processus implique une redéfinition des rapports entre les générations. L'allongement des carrières scolaires et l'élévation des cursus anticipés s'accompagnent d'un redécoupage des âges de la vie (avec notamment le développement d'un modèle estudiantin de la jeunesse ouvrière et le vieillissement précoce des parents ouvriers par la distance culturelle qui les éloigne de leurs enfants). Sans doute s'est-il ainsi creusé une certaine distance entre les générations ouvrières actuelles (Beaud et Pialoux, 1999).

Il n'est pas sûr, toutefois, qu'elle soit supérieure à celle qui a opposé les jeunes d'origine ouvrière nés dans les années 1940 à la génération de leurs parents (Terrail, 1991). Lorsqu'on interroge aujourd'hui les individus nés entre 1939 et 1943 sur leur perception des différences entre générations, ils estiment très majoritairement que celles qui les séparent de leurs enfants sont inférieures à celles qui les distinguent de leurs parents (nés dans les années 1910) (AttiasDonfut, 2000). En outre, ce clivage générationnel dans les familles ouvrières n'a pas anéanti une certaine continuité des aspirations entre les ouvriers d'aujourd'hui et leurs enfants. À bien des égards, les enfants d'ouvriers tentent de réaliser aujourd'hui ce dont leurs parents ont rêvé hier, dans un contexte il est vrai très différent. L'adoption par les parents comme par leurs enfants du modèle des études longues n'aurait ainsi pas été possible sans une appropriation des enjeux scolaires de génération en génération, qui peut s'expliquer par une adaptation aux transformations de l'institution scolaire d'une part (les ouvriers ont pris au sérieux l'idéologie méritocratique de l'école unique) et du marché de l'emploi d'autre part (les ouvriers n'ignorent pas le rôle de plus en plus déterminant des titres scolaires dans les recrutements professionnels). Si les familles ouvrières restent moins armées culturellement que les familles des classes supérieures dans cette course aux diplômes de plus en plus intensive, l'ampleur des changements d'attitude par rapport à l'école de génération en génération chez les ouvriers constitue bien une sorte de révolution culturelle qui n'a sans doute pas fini d'ébranler le système éducatif.

16. Les autres terrains de conflit proposés par le questionnaire étaient: la politique ou la religion, les vêtements et l'aspect physique, l'argent, les sorties, les fréquentations et les relations amoureuses, le choix du métier. 


\section{L'implication de la famille dans l'accès à l'emploi}

Q i l'avenir professionnel est de plus en plus fortement conditionné par la formation scolaire, il varie aussi selon le milieu familial d'origine, qui peut soutenir les jeunes dans leur accès au premier emploi. À diplôme égal, la réussite de l'insertion dépend encore notamment des relations sociales de la famille facilitant l'embauche (par le «piston» plus ou moins direct, l'introduction ou la recommandation auprès de l'employeur, le conseil avisé ou la circulation de l'information). Les jeunes non ou peu diplômés peuvent aussi bénéficier de cet appui pour obtenir par exemple un emploi saisonnier ou un stage (17). Relativement méconnues, ces stratégies familiales de valorisation ou de compensation du diplôme lors des premiers pas sur le marché du travail prennent une importance et des formes très variables selon les milieux sociaux. Contrairement à la thèse fonctionnaliste d'un affaiblissement du rôle économique de la famille d'origine dans les sociétés contemporaines (Parsons, 1955), plusieurs travaux récents ont ainsi souvent souligné, notamment dans les classes populaires, la persistance des liens de solidarité familiale et parfois remarqué leur rôle dans l'accès à l'emploi des jeunes générations (18).

\section{Le soutien familial perdure dans le monde ouvrier}

La parenté n'est donc pas morte, mais peu de matériaux permettent d'évaluer l'évolution historique de son importance sur le marché du travail. La progression inexorable du salariat, le recul de nombreux secteurs artisanaux, l'importance devenue primordiale des diplômes lors des embauches, l'accès à une plus grande autonomie des jeunes par rapport à leurs parents, etc., nombreux sont les faits sociaux allant dans le sens de la thèse du déclin de la fonction économique de la parenté. Les transformations du marché des emplois ouvriers pourraient également remettre en cause ce rôle d'intermédiaires des parents ouvriers. La progression du chômage ouvrier a en effet privé beaucoup de pères ouvriers de leurs réseaux de relations professionnelles mobilisables pour introduire leurs fils auprès d'employeurs potentiels : ainsi s'interpréterait la corrélation entre chômage des pères et difficultés d'insertion des fils (Marry, 1984).

Dans les années récentes, cette implication de la famille dans l'accès à l'emploi des jeunes peu qualifiés perdure cependant dans le monde ouvrier (Marry, 1992). L'enquête Transmissions familiales permet de le confirmer, d'indiquer quelques déterminants de cette forme de soutien et d'en évaluer l'impact sur les trajectoires des jeunes. $18 \%$ des enquêtés $(21 \%$ des enfants de cadres, $15 \%$ des enfants d'ouvriers) déclarent ainsi avoir reçu une aide parentale sur le plan professionnel. Près d'une fois sur deux, ceux-ci sont entrés dans l'entreprise où travaillaient leurs parents, et plus d'une fois sur deux, ils ont obtenu un travail grâce aux relations de leurs parents. Une infime minorité a bénéficié d'une aide financière des parents pour monter un projet. Qu'il s'agisse d'une recommandation auprès de l'employeur, ou encore d'une mise en relation visant à obtenir de l'information pour un emploi, l'appui des parents passe donc ici, avant tout, par la mobilisation de leurs réseaux de relations.

Mais vraisemblablement peu enclins à reconnaître le rôle du «piston » familial, contraire à l'esprit de la méritocratie, et sans doute plus oublieux, avec l'âge, des circonstances de leur propre accès à l'emploi, les individus interrogés sous-déclarent ce type de soutien. Une originalité de l'enquête est de les interroger aussi en tant que parents : $29 \%$ des enquêtés (27\% des ouvriers, $35 \%$ des cadres) disent alors avoir apporté une aide, sur le plan professionnel, à leurs enfants de plus de 18 ans. Doit-on dès lors conclure à une augmentation significative de l'engagement des parents dans les processus d'accès à l'emploi de leurs enfants ? La comparaison de l'aide apportée par les parents et de l'aide reçue par les enfants pour des mêmes cohortes d'enfants montre, d'une part, que l'aide déclarée par les parents est toujours supérieure à l'aide reconnue par les enfants (cf. graphique IV) (19) et, d'autre part, qu'il est difficile de dégager une tendance nette à la hausse. En outre, en milieu ouvrier, l'aide apportée aux enfants des cohortes nées entre 1945 et 1959 et entre 1960 et 1974 est stable (autour de 24 à $26 \%$ ). À l'aune de ces indica-

17. Beaucoup d'entreprises réservent par ailleurs ce type d'emplois aux enfants du personnel, qui constituent souvent pour eux une première étape vers l'embauche définitive. Voir par exemple (Desveaux, 1991).

18. Près d'un jeune de moins de 35 ans sur trois déclare ainsi avoir bénéficié d'un tel soutien de la famille. L'introduction auprès d'un employeur est plus fréquente dans les classes populaires: elle a concerné $26 \%$ des employés et $23 \%$ des ouvriers (quel que soit l'âge) (Bonvallet, Maison, Le Bras, et Charles, 1993). Voir aussi (Déchaux, 1990 ; Degenne, Fournier, Marry et Mounier, 1991 ; Attias-Donfut, Lapierre et Segalen, 2002).

19. C'est ainsi l'aide déclarée par les parents qui est retenue par la suite. 
teurs, les clivages «générationnels » n'apparaissent pas très évidents au sein du groupe ouvrier sur le plan du soutien familial pour l'accès à l'emploi.

\section{Une aide plus forte pour les moins diplômés...}

Si l'implication des familles dans les débuts de la trajectoire professionnelle de leurs enfants semble donc se maintenir dans le temps à travers ces stratégies d'accès à l'emploi, le sens de ces pratiques a sans doute évolué de génération en génération, sous l'effet de la double prolongation de la scolarisation et de la transition professionnelle. On peut penser que l'étirement de la période de passage entre la sphère éducative et la sphère productive permet et suscite un nouvel exercice de la solidarité familiale. De plus en plus nécessaire, le diplôme devient aussi de moins en moins suffisant pour garantir le succès de l'insertion professionnelle.

La gestion familiale des premiers pas des jeunes sur le marché de l'emploi varie ainsi selon leurs parcours scolaires. Les ouvriers ont apporté une aide sur le plan professionnel dans $30 \%$ des cas lorsque l'enfant détenait au plus un certificat d'études, un BEPC, un CAP ou un BEP. Cette aide n'a concerné que $22 \%$ des jeunes ayant décroché un diplôme du supérieur. Dans le premier cas, l'aide est sans doute facilitée par la proximité des parents avec ce type de formation et la connaissance de leurs débouchés. Dans le

\section{Graphique IV}

Aide des parents sur le plan professionnel

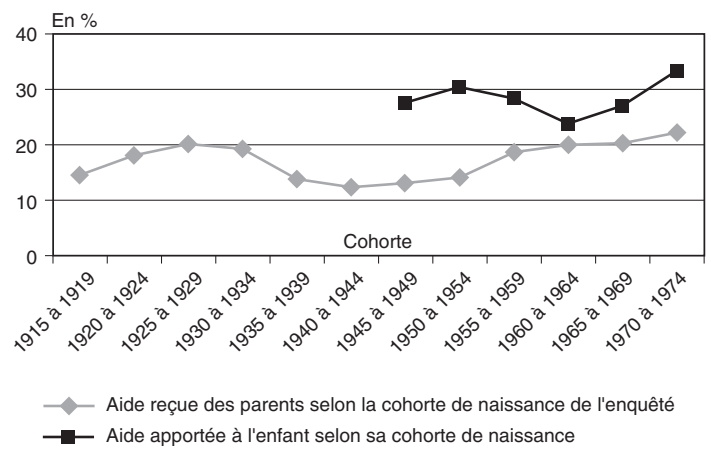

Lecture : pour chaque aide, on a construit un indicateur signalant au moins l'une des trois aides suivantes : entrée dans l'entreprise où travaillait le parent, embauche par les relations du parent, aide financière pour monter un projet. Un peu plus de $30 \%$ des parents d'enfants nés entre 1950 et 1974 leur ont apporté une aide au plan professionnel.

Champ: pour l'aide apportée à l'enfant, personnes ayant un enfant de plus de 18 ans non scolarisé ; pour l'aide reçue des parents, personnes de plus de 15 ans.

Source : enquête Transmissions familiales, Insee, 2000. second cas, au contraire, la moindre familiarité avec l'univers de l'enseignement supérieur fait davantage reposer la stratégie d'accès à l'emploi sur les jeunes eux-mêmes (20). L'enquête ne permet pas de décrire beaucoup mieux l'évolution de ces stratégies de génération en génération. Elle permet, en revanche, d'examiner certains aspects de l'efficacité ou de l'impact de l'aide apportée aux enfants sur le plan professionnel en termes de mobilité sociale.

\section{... qui tend à maintenir dans le milieu d'origine}

À quels types d'emplois peuvent donc conduire ces aides apportées par les parents ? Hormis les indépendants (agriculteurs, artisans, commerçants, chefs d'entreprise) et les professions libérales, où l'aide familiale intense (notamment financière) est liée à une logique d'accumulation ou de transmission patrimoniale, les catégories du salariat qui ont le plus souvent bénéficié de ces efforts familiaux sont les ouvriers et les employés administratifs d'entreprise. Si l'on s'intéresse maintenant aux destinées sociales des enfants qui ont été aidés professionnellement par leurs parents, on constate certaines différences par rapport à ceux qui n'ont pas été aidés (cf. tableau 6) (21).

Mis à part ceux qui ont été aidés financièrement pour monter un projet, pour lesquels ce soutien a permis l'accès à une profession indépendante bien plus souvent que pour les autres, la mobilisation des ressources relationnelles ne change pas beaucoup la donne, si ce n'est qu'elle augmente les chances de rester dans les catégories populaires pour les enfants d'agriculteurs, d'employés ou d'ouvriers. En particulier, parmi les enfants d'ouvriers (22), ceux qui ont bénéficié de l'aide d'un de leurs parents sont plus souvent classés ouvriers que les autres (36\% contre $28 \%$ ). Ils ont, en revanche, des chances à peu près égales, qu'ils aient été aidés ou non, de

20. Parmi les étudiants, un enfant d'ouvrier sur trois seulement pense que son père comprend le genre d'études qu'il fait, qu'il a une idée précise de ce qu'elles signifient (contre sept enfants de cadres sur dix). Moins d'un sur six a eu des informations utiles pour son insertion professionnelle de la part de son père (contre plus d'un sur deux pour les enfants de cadres). Ces résultats sont tirés de l'exploitation de l'enquête Efforts d'éducation des familles réalisée par l'Insee et l'Ined en 1992.

21. On ne s'intéresse par la suite qu'à deux formes d'aides, qu'on a regroupées dans un seul indicateur: l'entrée dans l'entreprise où travaillait un des parents et l'aide pour trouver du travail grâce aux relations des parents.

22. Le milieu social où a grandi l'enfant est repéré par la catégorie socioprofessionnelle de la personne de référence du ménage, et non par celle du répondant. 
devenir cadres moyens ou supérieurs. Ces résultats confortent les travaux de Granovetter sur la rentabilité différentielle des réseaux de relations mobilisés pour obtenir un emploi (Granovetter, 1973) : contrairement à l'utilisation des liens faibles, le recours aux relations familiales n'améliore pas les chances d'accéder aux meilleurs emplois. L'enquête Transmissions familiales montre, au contraire, qu'elle renforce les risques de rester dans leur milieu d'origine pour les enfants d'ouvriers. Sans doute la distance sociale entre les familles ouvrières et les classes supérieures ne leur permet-elle pas de mobiliser des contacts sociaux débouchant sur des emplois d'encadrement. On peut penser aussi que l'implication de la famille dans la recherche d'emploi relève d'une stratégie défensive pour compenser un capital scolaire faible des enfants dans une conjoncture économique dégradée.

\section{Soutien familial et ascension sociale ne vont pas de pair pour les enfants d'ouvriers}

On peut aussi mesurer l'efficacité de l'aide apportée par les parents en termes de mobilité sociale en contrôlant d'autres facteurs (cf. graphiques V, VI et VII). Il s'agit ici de vérifier le rôle de l'origine sociale, du diplôme ou du sexe dans l'étude de la mobilité sociale : les enfants de cadres, les garçons et les plus diplômés sont les mieux placés pour atteindre des positions de cadre ou de professions intermédiaires. Les cohortes les plus anciennes connaissent aussi des chances supérieures d'accéder aux positions d'encadrement et d'éviter les catégories populaires, sans qu'on puisse démêler ici les inégalités liées à la date de naissance (Chauvel, 1998) et le rôle des carrières professionnelles (inégalement achevées selon l'âge à l'enquête). Mais, toutes choses égales par ailleurs, le modèle montre aussi un effet significatif de l'aide parentale sur le plan professionnel concernant les destinées ouvrières pour les enfants. Parmi les enfants d'ouvriers, ceux qui ont été aidés sont sensiblement plus souvent ouvriers que les autres.

Il apparaît difficile finalement de savoir si l'aide parentale apportée aux enfants sur le plan professionnel relève de «l'aide pour la promotion » ou de «l'aide pour la

Tableau 6

Destin social des enfants selon l'aide apportée par les parents sur le plan professionnel

Réponse à la question : "Quelle est la situation professionnelle actuelle de votre enfant ou la dernière qu'il ait eue ? "

En \% de réponses

\begin{tabular}{|c|c|c|c|c|c|c|}
\hline \multirow[b]{2}{*}{$\begin{array}{l}\text { Catégorie } \\
\text { socioprofessionnelle } \\
\text { de la personne de } \\
\text { référence du ménage }\end{array}$} & \multirow[b]{2}{*}{$\begin{array}{l}\text { Aide apportée } \\
\text { par les parents } \\
\text { sur le plan } \\
\text { professionnel }\end{array}$} & \multicolumn{5}{|c|}{ Catégorie socioprofessionnelle de l'enfant } \\
\hline & & $\begin{array}{c}\text { Agriculteurs, } \\
\text { artisans, } \\
\text { commerçants et } \\
\text { chefs d'entreprise }\end{array}$ & $\begin{array}{c}\text { Cadres et professions } \\
\text { intellectuelles } \\
\text { supérieures, } \\
\text { professions } \\
\text { intermédiaires }\end{array}$ & Employés & Ouvriers & Ensemble \\
\hline \multirow{3}{*}{$\begin{array}{l}\text { Agriculteurs, artisans, } \\
\text { commerçants et chefs } \\
\text { d'entreprise }\end{array}$} & Non & 14 & 34 & 38 & 14 & 100 \\
\hline & Oui & 37 & (24) & (23) & $(16)$ & 100 \\
\hline & Ensemble & 21 & 31 & 33 & 15 & 100 \\
\hline \multirow{3}{*}{$\begin{array}{l}\text { Cadres et professions } \\
\text { intermédiaires }\end{array}$} & Non & (4) & 58 & 31 & (7) & 100 \\
\hline & Oui & (3) & 43 & 44 & (10) & 100 \\
\hline & Ensemble & (3) & 53 & 35 & 8 & 100 \\
\hline \multirow{3}{*}{ Employés } & Non & (4) & 41 & 40 & 14 & 100 \\
\hline & Oui & (3) & (29) & 46 & (22) & 100 \\
\hline & Ensemble & (4) & 39 & 42 & 16 & 100 \\
\hline \multirow{3}{*}{ Ouvriers } & Non & (5) & 22 & 45 & 28 & 100 \\
\hline & Oui & (4) & (21) & 38 & 36 & 100 \\
\hline & Ensemble & (5) & 22 & 43 & 30 & 100 \\
\hline \multirow{3}{*}{ Ensemble } & Non & 6 & 38 & 39 & 17 & 100 \\
\hline & Oui & 11 & 30 & 38 & 21 & 100 \\
\hline & Ensemble & 7 & 36 & 39 & 18 & 100 \\
\hline
\end{tabular}

Lecture : $30 \%$ des enfants d'ouvriers sont devenus ouvriers. Cette proportion passe à $36 \%$ lorsqu'ils ont été aidés par leurs parents pour accéder à un emploi. Les parenthèses signalent des pourcentages fragiles du fait de petits effectifs. L'aide dont il est question ici est soit l'entrée dans l'entreprise où travaillait un des parents, soit l'obtention d'un travail par les relations des parents.

Champ : personnes ayant un enfant de plus de 18 ans qui a terminé ses études ou n'en a jamais fait (échantillon de la table des transmissions effectuées).

Source : enquête Transmissions familiales, Insee, 2000. 
Graphique V

Modélisation du taux d'accès des enfants aux catégories de cadres ou de professions

intermédiaires

Situation de référence en italique

CSP de la personne de référence du ménage

Ouvrier

Agriculteur ou indépendant

Employé

Cadre ou profession intermédiaire

Sexe de l'enquêté

Femme

Homme

Niveau d'étude de l'enfant

Diplôme inférieur au bac

Diplôme supérieur ou égal au bac

Aide des parents pour accéder à un emplo

Non

Oui

Cohorte de naissance de l'enfant

1945-1954

1955-1964

1965-1974

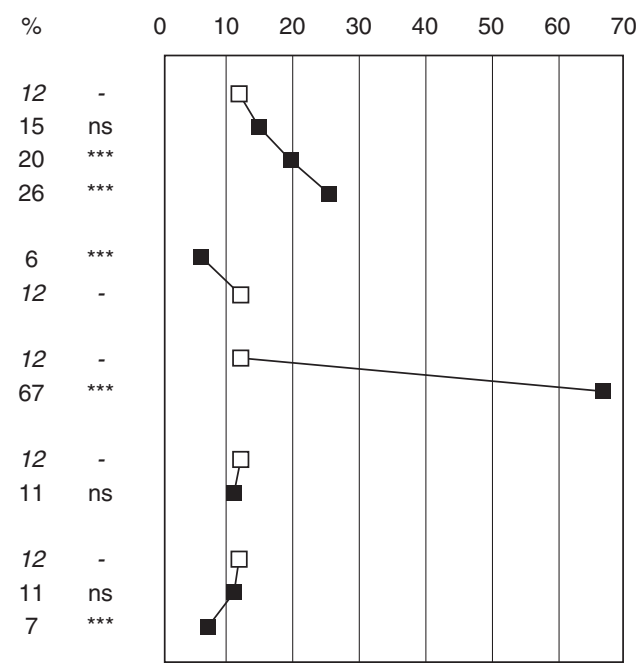

Lecture : il s'agit d'un modèle de type logit. On part d'une situation de référence signalée en italique : un fils né entre 1945 et 1954 de parents ouvriers, qui possède un diplôme inférieur au bac et n'a pas été aidé par ses parents pour accéder à un emploi a $12 \%$ de chances d'être cadre ou profession intermédiaire en 2000. Le modèle permet d'évaluer qu'un enfant de cadre ou de profession intermédiaire présentant par ailleurs toutes les caractéristiques de cette situation de référence aurait $26 \%$ de chances d'occuper la même position. L'écart des chances (14 points) mesure ainsi l'effet propre de l'origine sociale sur les chances d'accès aux catégories de cadres ou de professions intermédiaires. Dans la seconde colonne, ${ }^{* *}$ indique que le paramètre estimé est significatif au seuil de $5 \%{ }^{* * *}$ pour un seuil de $1 \%$, * pour un seuil de $15 \%$, ns pour un test non significatif).

Champ : enfants nés entre 1945 et 1974, élevés par leurs parents, ayant suivi des études. Les non-réponses ont été enlevées de l'échantillon pour la régression logistique (échantillon de la table des transmissions effectuées, $N=1567$ ).

Source : enquête Transmissions familiales, Insee, 2000

Graphique VI

Modélisation du taux d'accès des enfants aux catégories ouvrières

Situation de référence en italique

CSP de la personne de référence du ménage

Agriculteur ou indépendant

Cadre ou profession intermédiaire

Employé

Ouvrier

Sexe de l'enquêté

Femme

Homme

Niveau d'étude de l'enfant

Diplôme inférieur au bac

Diplôme supérieur ou égal au bac

Aide des parents pour accéder à un emploi

Non

Oui

Cohorte de naissance de l'enfant

1945-1954

1955-1964

$1965-1974$

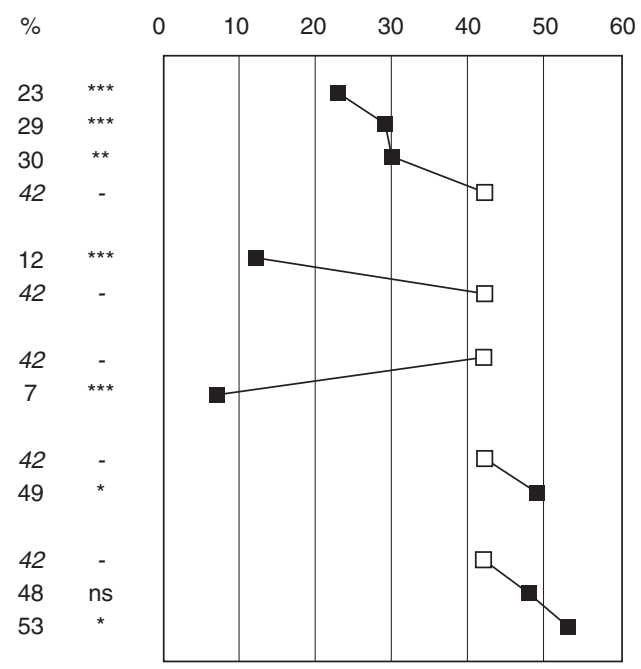

Lecture : il s'agit d'un modèle de type logit. On part d'une situation de référence signalée en italique : un fils né entre 1945 et 1954 de parents ouvriers, qui possède un diplôme inférieur au bac et n'a pas été aidé par ses parents pour accéder à un emploi a 42 \% de chances d'être ouvrier en 2000. Le modèle permet d'évaluer qu'un enfant ayant été aidé par ses parents pour accéder à un emploi et présentant par ailleurs toutes les caractéristiques de cette situation de référence aurait $49 \%$ de chances d'occuper la même position. L'écart des chances (7 points) mesure ainsi l'effet propre de l'aide des parents pour obtenir un emploi sur les chances d'accès aux catégories ouvrières. Dans la seconde colonne, ${ }^{* *}$ indique que le paramètre estimé est significatif au seuil de $5 \%\left(^{* * *}\right.$ pour un seuil de $1 \%$, ${ }^{*}$ pour un seuil de $15 \%$, ns pour un test non significatif).

Champ : enfants nés entre 1945 et 1974, élevés par leurs parents, ayant suivi des études. Les non-réponses ont été enlevées de l'échantillon pour la régression logistique (échantillon de la table des transmissions effectuées, $N=1567$ ).

Source : enquête Transmissions familiales, Insee, 2000. 
subsistance » (23), s'il s'agissait dans l'esprit des intéressés d'obtenir un emploi avec l'espoir d'y faire carrière ou simplement d'éviter le chômage et la précarité, le questionnaire donnant peu d'informations sur l'insertion professionnelle des jeunes. L'enquête confirme, en revanche, que l'accès à l'emploi par le biais de ces médiations familiales ne va pas de pair avec une mobilité intergénérationnelle ascendante (Marry, 1992). Dans les familles ouvrières, elle semble contribuer, au contraire, à la reproduction de la position sociale d'une génération à l'autre (24). Au total, le maintien à travers les générations ouvrières du recours aux solidarités familiales pour aider les jeunes à pénétrer le marché du travail reste ambivalent. Ces pratiques attestent les capacités traditionnelles de mobilisation familiale chez les ouvriers mais leur rentabilité sociale sur le marché du travail contemporain est incertaine. Elles perdurent malgré les profondes transformations de l'appareil productif et, plus largement, de la société française, mais bien plus pour compenser des diplômes de plus en plus dévalorisés aujourd'hui que pour ajouter la valeur du «capital social» des parents aux diplômes de l'enseignement supérieur obtenus par les enfants.

\section{Des transmissions familiales désormais centrées sur la scolarisation}

Étudiées sous l'angle de la succession et du renouvellement des générations, les transformations du groupe ouvrier peuvent ainsi faire apparaître utilement certaines ruptures intergénérationnelles dans les pratiques ou les aspirations des ouvriers et de leurs enfants de nature à expliquer les nouvelles modalités des transmissions familiales. Si les inégalités scolaires restent massives depuis une quarantaine d'années, des évolutions importantes sont à l'œuvre dans le rapport des ouvriers à l'école. Entre les années 1960 et les années 1990, les ouvriers ont ainsi changé fondamentalement de comportement face à la scolarité et aux trajets sociaux de leurs enfants : tendanciellement, ils sont passés d'une relative extériorité par rapport à l'école (nos enfants y feront ce qu'ils pourront) à une préoccupation massive pour la réussite scolaire (nos enfants doivent continuer

23. Selon les catégories élaborées par (Pitrou, 1992).

24. On peut supposer cependant que l'aide pour la promotion sociale des enfants passe dans les familles ouvrières par d'autres échanges, comme les aides pour le logement, l'entraide familiale pour la garde des enfants, etc. (Pitrou, 1992).

\section{Graphique VII \\ Modélisation du taux d'accès des enfants à la catégorie des employés}

Situation de référence en italique

CSP de la personne de référence du ménage

Agriculteur ou indépendant

Cadre ou profession intermédiaire

Ouvrier

Employé

Sexe de l'enquêté

Femme

Homme

Niveau d'étude de l'enfant

Diplôme inférieur au bac

Diplôme supérieur ou égal au bac

Aide des parents pour accéder à un emploi

Non

Oui

Cohorte de naissance de l'enfant

1945-1954

$1955-1964$

$1965-1974$

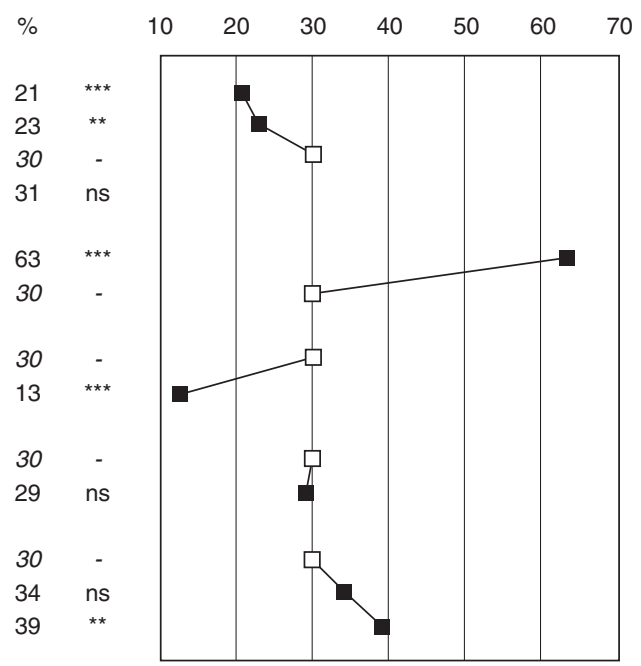

Lecture : il s'agit d'un modèle de type logit. On part d'une situation de référence signalée en italique : un fils né entre 1945 et 1954 de parents ouvriers, qui possède un diplôme inférieur au bac et n'a pas été aidé par ses parents pour accéder à un emploi a $30 \%$ de chances d'être ouvrier en 2000. Le modèle permet d'évaluer qu'un enfant ayant au moins le bac et présentant par ailleurs toutes les caractéristiques de cette situation de référence aurait $13 \%$ de chances d'occuper la même position. L'écart des chances (17 points) mesure ainsi l'effet propre du niveau d'étude sur les chances d'accès à la catégorie des employés. Dans la seconde colonne, ${ }^{\star *}$ indique que le paramètre estimé est significatif au seuil de $5 \%$ (** pour un seuil de $1 \%$, * pour un seuil de $15 \%$, ns pour un test non significatif).

Champ : enfants nés entre 1945 et 1974, élevés par leurs parents, ayant suivi des études. Les non-réponses ont été enlevées de l'échantillon pour la régression logistique (échantillon de la table des transmissions effectuées, $N=1567$ ).

Source : enquête Transmissions familiales, Insee, 2000. 
le plus loin possible leurs études pour pouvoir faire ce qu'ils voudront dans la vie) (25).

Loin de réduire les ambitions scolaires plus élevées des nouvelles générations à des illusions anomiques, l'enquête Transmissions familiales atteste une adaptation active au nouveau poids du diplôme dans les destinées sociales, qui implique un bouleversement des stratégies éducatives familiales désormais centrées sur la scolarisation. La permanence d'une certaine implication des familles ouvrières, de génération en génération, dans la gestion de l'insertion professionnelle de leurs enfants indique, par ailleurs, que les métamorphoses de la société française n'invalident pas totalement les stratégies de mobilisation des ressources relationnelles, même si elles contribuent davantage à perpétuer la position sociale de la lignée qu'à favoriser la mobilité ascendante des enfants.

Cette approche permet ainsi de caractériser un groupe social non seulement par son passé et ses héritages mais aussi par la façon dont ses membres se projettent dans le futur, de mieux comprendre ainsi son identité temporelle. À cet égard, on ne saurait non plus surestimer le fossé qui semble se creuser entre les générations ouvrières (26). Si profond qu'il paraisse aujourd'hui, il n'annule pas toutes les continuités qui se font jour à travers les transmissions intergénérationnelles et les solidarités familiales. Les rapports qui se nouent entre les générations ouvrières autour de la scolarisation et de l'insertion professionnelle des enfants portent, certes, la spécificité de chaque génération, mais ils contribuent en retour à les modeler l'une par rapport à l'autre. Leur histoire a ainsi partie liée avec le changement social: les interactions entre générations peuvent l'initier ou l'empêcher, l'accélérer ou l'amortir (Attias-Donfut, 2000).

25. Dans leur enquête réalisée à Amiens en 1980, Desrosières et Gollac (1981) insistaient déjà sur l'opposition sociale entre ces deux conceptions de l'existence chez les parents : « alors que la première, caractéristique des classes populaires et moyennes, fait référence à ce que l'enfant pourra, la seconde, plus répandue dans les classes supérieures, s'en remet à ce que l'enfant voudra. "Quelques années plus tard, enquêtant sur les élèves de LEP d'origine populaire à Nantes, Baudelot et Establet signalent que ceux qui ont connu les meilleurs parcours scolaires avant d'entrer dans les filières les plus sélectives de CAP ou de BEP se distinguent par une conception de la vie réussie où prime l'idéal de "faire ce que l'on veut, ce qui plaît " (Baudelot et Establet, 1989, pp. 131-146).

26. On sait que plus les parents vieillissent, plus ils sont enclins à percevoir la distance avec leurs enfants. Halbwachs souligne ainsi que « suivant l'âge et aussi les circonstances, on est frappé surtout des différences ou des similitudes entre les générations qui tantôt se replient sur elles-mêmes et s'éloignent l'une de l'autre et tantôt se rejoignent et se confondent. " (Halbwachs, 1950).

\section{BIBLIOGRAPHIE}

Attias-Donfut C. (1988), Sociologie des générations. L'empreinte du temps, Paris, PUF.

Attias-Donfut C. (2000), « Rapports de générations. Transferts intrafamiliaux et dynamique macrosociale », Revue française de sociologie, $n^{\circ}$ 41-4, pp. 643-684.

Attias-Donfut C., Lapierre N. et Segalen M. (2002), Le nouvel esprit de famille, Paris, Éditions Odile Jacob.

Baudelot C. et Gollac M. (dir.) (2003), Travailler pour être heureux? Le bonheur et le travail en France, Paris, Éditions Fayard.

Baudelot C. et Gollac M. (1997), « Le salaire du trentenaire : question d'âge ou de génération? », Économie et Statistique, $\mathrm{n}^{\circ}$ 304-305, pp. 17-36.
Beaud S. (2002), $80 \%$ au bac... et après? Les enfants de la démocratisation scolaire, Paris, Éditions La Découverte.

Beaud S. et Pialoux M. (1999), Retour sur la condition ouvrière. Enquête aux usines Peugeot de Sochaux - Montbéliard, Paris, Éditions Fayard.

Bonvallet C., Maison D., Le Bras H. et Charles L. (1993), « Proches et parents », Population, $\mathrm{n}^{\circ}$ 1, pp. 83-109.

Boudon R. (1973), L'inégalité des chances, Paris, Éditions A. Colin.

Chauvel L. (1998), Le destin des générations. Structure sociale et cohortes en France au $X X^{e}$ siècle, Paris, PUF. 
Déchaux J.-H. (1990), «Échanges économiques et parentèles », Sociologie du travail, $\mathrm{n}^{\circ} 1$, pp. 7394.

Degenne A., Fournier I., Marry C. et Mounier L. (1991), «Les relations sociales au cœur du marché du travail », Sociétés contemporaines, $\mathrm{n}^{\circ} 5$, pp. 75-97.

Desveaux E. (1991), « De l'embauche à l'usine comme de la dévolution d'un patrimoine », in : Segalen M. (éd.), Jeux de familles, Paris, Éditions du CNRS, pp. 43-56.

Granovetter M. (1973), « The Strength of Weak Ties », American Journal of Sociology, 78 (6), pp. 1360-1380 (traduit dans Granovetter M. (2000), Le marché autrement, Paris, Éditions Desclée de Brouwer).

Halbwachs M. (1950), La mémoire collective, Paris, PUF.

Ined (1970), Population et l'enseignement, Paris, PUF.

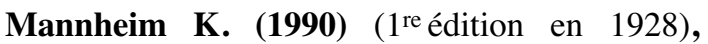
Le problème des générations, Paris, Éditions Nathan.

Marry C. (1984), «Origine sociale et réseaux d'insertion des jeunes ouvriers », Formation Emploi, ${ }^{\circ} 4$, pp. 3-15.

Marry C. (1992), « Les jeunes et l'emploi. Force et faiblesse des liens forts », in Coutrot L. et Dubar C. (éds), Cheminements professionnels et mobilités sociales, Paris, La documentation Française, pp. 300-324.
Mauger G. (1998), « La reproduction des milieux populaires en crise », Ville - École - Intégration, $\mathrm{n}^{\circ} 113$, pp. 6-16.

Minonzio J. (2000), « Solidarités familiales et chômage », Recherches et prévisions, $\mathrm{n}^{\circ} 60$, pp. 35-47.

Mounier L. (1999), « À quoi peuvent servir les relations sociales des jeunes? », Agora, $\mathrm{n}^{\circ}$ 17, pp. 47-62.

Noiriel G. (1986), Les ouvriers dans la société française, Paris, Éditions du Seuil.

Parsons T. (1955), ( $1^{\text {re }}$ parution aux États-Unis en 1943), Le système de parenté dans les États-Unis d'aujourd'hui. Éléments pour une sociologie de l'action, Paris, Éditions Plon.

Pitrou A. (1992), Les solidarités familiales. Vivre sans famille?, Toulouse, Éditions Privat.

Terrail J.-P. (1984), « Familles ouvrières, école, destin social (1880-1980) », Revue française de sociologie, $\mathrm{n}^{\circ} 25-3$, pp. 421-436.

Terrail J.-P. (1990), Destins ouvriers. La fin d'une classe?, Paris, PUF.

Terrail J.-P. (1991), «Les générations sociales dans l'après-guerre », Annales de Vaucresson, $\mathrm{n}^{\circ}$ 30-31, pp. 105-126.

Terrail J.-P. (1992), « Destins scolaires de sexe : une perspective historique et quelques arguments », Population, n 3 , pp. 645-676.

Verret M. (1992), « Où va la classe ouvrière française ? », in « Ouvriers, ouvrières. Un continent morcelé et silencieux », Autrement, $\mathrm{n}^{\circ} 126$, pp. 21-33. 